\title{
Identification of the DNA Replication Regulator MCM Complex Expression and Prognostic Significance in Hepatic Carcinoma
}

\author{
Ting Cao, ${ }^{1}$ Shi-jie Yi, ${ }^{2}$ Li-xin Wang, ${ }^{3}$ Juan-xia Zhao, ${ }^{4}$ Jiao Xiao, ${ }^{5}$ Ni Xie, ${ }^{1}$ Zhi Zeng, ${ }^{6}$ Qi Han, \\ Hai-ou Tang, ${ }^{8}$ Yu-kun Li ${ }^{1},{ }^{9}$ Juan Zou ${ }^{D},{ }^{9}$ and Qing Wu ${ }^{1}{ }^{1}$ \\ ${ }^{1}$ Department of Digestive Medical, The Affiliated Nanhua Hospital, University of South China, Hengyang 421002, China \\ ${ }^{2}$ Department of Gastrointestinal Surgery, The Affiliated Nanhua Hospital, University of South China, Hengyang 421002, China \\ ${ }^{3}$ Center for Traditional Chinese Medicine and Immunology Research, School of Basic Medical Sciences, Shanghai University of \\ Traditional Chinese Medicine, 1200 Cai Lun Rd., Shanghai 201203, China \\ ${ }^{4}$ Department of Pathology, The Affiliated Nanhua Hospital, University of South China, Hengyang 421002, China \\ ${ }^{5}$ Department of Endocrinology, The Affiliated Nanhua Hospital, University of South China, Hengyang 421002, China \\ ${ }^{6}$ Department of Pathology, Xianning Central Hospital, The First Affiliated Hospital of Hubei University of Science and Technology, \\ Xianning 437000, China \\ ${ }^{7}$ Department of Oncology, Xianning Central Hospital, The First Affiliated Hospital of Hubei University of Science and Technology, \\ Xianning 437000, China \\ ${ }^{8}$ Jishou University College of Medicine, Jishou 416000, China \\ ${ }^{9}$ Key Laboratory of Tumor Cellular and Molecular Pathology, College of Hunan Province, Cancer Research Institute, University of \\ South China, Hengyang, Hunan 421001, China
}

Correspondence should be addressed to Juan Zou; zoujuangzy@163.com and Qing Wu; nhwuqing@163.com

Received 28 March 2020; Revised 8 August 2020; Accepted 12 August 2020; Published 9 September 2020

Academic Editor: Elena Orlova

Copyright $\odot 2020$ Ting Cao et al. This is an open access article distributed under the Creative Commons Attribution License, which permits unrestricted use, distribution, and reproduction in any medium, provided the original work is properly cited.

Background. The microliposome maintenance (MCM) complex, MCM2-7, is revealed to be involved in multiple cellular processes and plays a key role in the development and progression of human cancers. However, the MCM complex remains poorly elaborated in hepatic carcinoma (HCC). Methods. In the study, we found the mRNA and protein level by bioinformatics. We also explored the prognostic value, genetic alteration, interaction network, and functional enrichment of MCM2-7. The MCM expression and correlation among these MCMs in HCC cell lines were identified by western blot. Results. MCM2-7 was significantly increased in HCC tissues compared to normal liver tissues. The high level of MCM2-7 had a positive correlation with poor prognosis. However, MCM2-7 alterations were not correlated with poor OS. MCMs were both increased in HCC cell lines compared to the normal hepatocyte cell line. Furthermore, the positive correlation was found among MCMs in HCC cell lines. Conclusions. The MCM complex was increased in HCC tissues and cell lines and negatively correlated with prognosis, which might be important biomarkers for HCC.

\section{Introduction}

Hepatic carcinoma (HCC), a severe malignant disease of the digestive system, ranks sixth in terms of morbidity (over 0.8 million new cases) and fourth in mortality overall (over 0.7 million deaths) in 2018 [1]. There are two important risk factors for HCC, including alcohol consumption [2] and hepatitis virus [3]. Although the development of new targeted drugs and multidrug combinations has improved the battlefield for HCC, only about 5-14 percent of patients with HCC have a five-year survival rate $[4,5]$, with the development of bioinformatics and high-throughput sequencing technology, inhibitor of apoptosis protein (IAP) family members [6], kinesin family members [6], cytochrome P2C (CYP2C) subfamily members [7], CDK1, PBK, RRM2, and ASPM [8]. Recently, Han et al. found through bioinformatics that 
SCAMP3 may be an important marker in the development of liver cancer [9]. Therefore, it is very important to explore the correlation between prognostic value and new gene complexes in HCC.

The microliposome maintenance (MCM) protein was first discovered in sacs cerevisiae, and the mutants showed defects in microliposome maintenance, which play a key role in DNA replication [10]. MCM2-7 is a group of six structurally related proteins, from yeast to humans, which are highly conserved and interact to form a hexamer. In the progression of DNA synthesis, MCM2-7, as nuclear proteins, binds to chromatin by a cell cycle specific manner, resulting in promoting cell proliferation and helicase activity [10]. Disorders in these proteins can directly disrupt the DNA replication system, leading to cancer occurrence, development, and progression [11].

The MCM complex acts as an important regulator in multiple pathophysiological processes, including DNA replication [12], cell cycle [11], proliferation [13], migration [14], invasion [14], immune response [15], and apoptosis [11]. It has been previously reported in the literature that MCM is highly expressed in many cancers compared to normal tissues. For example, MCM2 got a high level in HCC, oral squamous cell carcinoma [16], gastric cancer [17], breast cancer [18], colon cancer [19], and ovarian cancer [20]. MCM2 was considered as a potential therapeutic target for cancer treatment, and the level of MCM2 could predict poor prognosis for osteosarcoma [21], gastric cancer [22], lung adenocarcinoma [23], diffuse large B cell lymphoma [24], and esophageal cancer [25]. Recent research suggested that MCM2 might be a potential therapeutic target for HCC [26]. Furthermore, Deng et al. found that MCM2 inhibition could increase the sensitivity of carboplatin in ovarian cancer cell [27]. MCM3 had similarly a high expression level in multiple cancer types, such as osteosarcoma [21], salivary gland tumors [28], and glioma [29]. Ha et al. indicated that MCM3 got a high expression in leukemia, lymphoma, uterine cervix cancer, colon cancer, lung cancer, gastric cancer, kidney cancer, breast cancer, and malignant melanoma [30]. MCM4 levels were elevated in esophageal cancer [31], uterine cervical carcinoma [32], and non-small-cell lung cancer [33]. MCM5, as another regulator in DNA replication, was overexpressed in colon cancer [19], oral squamous cell carcinoma [34], cervical cancer [35], thyroid cancer [36], and bladder cancer [37]. The expression of MCM6 was found to be enhanced and its high level had a close relationship with unfavorable prognosis in colorectal cancer [38], breast cancer [39], AO [15], HCC [14], endometrioid adenocarcinoma [40], lung cancer [41], meningiomas [42], cervical cancer [43], Hodgkin's lymphoma [44], and Merkel cell carcinoma [45]. Ectopic expression of MCM7 has been indicated that can promote the progression of prostate cancer [46], HCC [47], breast cancer [48], and acute myeloid leukemia [49].

These studies sufficiently indicated differential level of MCMs in multiple cancer types, but few studies systematically focused on the prognostic value of the whole MCM complex members in carcinogenesis. In this study, we comprehensively demonstrated the transcriptional level of MCMs and found its prognostic value in HCC. Moreover, we also analyzed the interaction network, genetic mutation, and functional enrichment of MCMs by bioinformatics.

\section{Materials and Methods}

2.1. Oncomine Analysis. Oncomine (https://www.oncomine .org) is a free access website to facilitate genome-wide expression analysis. We analyzed the transcriptional levels of MCM information in HCC from the Oncomine database [50].

2.2. GEPIA Analysis. Gene Expression Profiling Interactive Analysis (GEPIA) (http://gepia.cancer-pku.cn/), based on TCGA and GTEx data, is a web-based tool that delivers rapid customization to evaluate the relationship between MCM expression and staging in HCC [51].

2.3. Human Protein Atlas Analysis. The Human Protein Atlas (HPA) (https://www.proteinatlas.org/), an open online database of protein expression profiles, assists researchers in studying the differential expression of proteins between cancer and normal tissues.

2.4. Clinical Samples. A total of 30 HCC tissues were surgically resected in the Affiliated Nanhua Hospital, University of South China (Hengyang, Hunan, China), from 2010 to 2014. These tissues were made to 3 pieces of $10 \times 10$ chips. The collection and use of tissues followed the procedures according to the ethical standards as formulated in the Helsinki Declaration. And written informed consent was obtained from each patient, which was approved by the research ethics committee of University of South China. All patients did not receive radiotherapy or chemotherapy.

2.5. Kaplan-Meier Plotter Analysis. KM plotter (http:// kmplot.com/analysis/), a survival database of patients, can analyze survival curves for many types of cancer [6]. The $\mathrm{KM}$ plotter is utilized to assess the prognostic value of MCMs in HCC.

2.6. GeneMANIA and STRING Analysis. GeneMANIA (http://genemania.org) [52] and STRING (https://string-db .org/) [53] are web tools to identify the interactions between genes/proteins, respectively. The interactions between MCMs and other gene/proteins are utilized by GeneMANIA and STRING at the gene or protein level.

2.7. cBioPortal for Cancer Genomics Analysis. cBioPortal for Cancer Genomics (http://www.cbioportal.org/), an open and free web tool, can be used to interactively explore multiple cancer genome datasets [54]. The correlation between MCM alterations and survival outcome in patients with HCC was analyzed by cBioPortal.

2.8. Metascape Analysis. Metascape (http://metascape.org) is an online analysis website available at enrichment pathway analysis and gene function annotation, which can be used to analyze the pathway and process enrichment of MCMs and the 40 neighboring genes [55].

2.9. Cell Culture. Five human HCC cell lines (HepG2, SNU354, Huh 7, SNU-739, and HLF) and a normal human liver 
cell line (HL-7702) were cultured in Dulbecco's Modified Eagle Medium (DMEM; Gibco) containing 10\% fetal bovine serum (FBS), $100 \mathrm{U} / \mathrm{mL}$ penicillin, and streptomycin, maintained at $37^{\circ} \mathrm{C}$ in a humidified atmosphere containing $5 \%$ $\mathrm{CO}_{2}$.

2.10. Quantitative Reverse Transcription Polymerase Chain Reaction (PCR). PCR was conducted as previously described. Primers used were listed as follows: GAPDH forward GTCTCC TCTGACTTCAACAGCG, GAPDH reverse ACCACCCTG TTGCTGTAGCCAA; MCM2 forward TGCCAGCATTG CTCCTTCCATC, MCM2 reverse AAACTGCGACTTCGCT GTGCCA; MCM3 forward CGAGACCTAGAAAATGGCA GCC, MCM3 reverse GCAGTGCAAAGCACATACCGCA; MCM4 forward CTTGCTTCAGCCTTGGCTCCAA, MCM4 reverse GTCGCCACACAGCAAGATGTTG; MCM5 forward GACTTACTCGCCGAGGAGACAT, MCM5 reverse TGCT GCCTTTCCCAGACGTGTA; MCM6 forward GACAAC AGGAGAAGGGACCTCT, MCM6 reverse GGACGCTTT ACCACTGGTGTAG; and MCM7 forward GCCAAGTCT CAGCTCCTGTCAT, MCM7 reverse CCTCTAAGGTCAGT TCTCCACTC.

2.11. Immunohistochemistry. According to the manufacturer's instructions (Maixin Biotech. Co., Fuzhou, China), the slides were incubated with the primary antibody (diluted $1: 100)$ at $4^{\circ} \mathrm{C}$ overnight, and normal rabbit immunoglobulin $\mathrm{G}$ was the negative control. The score of positive staining degree and percentage of stained cells were as follows: 0 , no staining; 1, light brown; 2, dark brown and 0 , stained cells $<5 \%$; 1 , stained cells range from $5 \%$ to $25 \%$; 2 , stained cells ranged from $26 \%$ to $50 \%$; 3 , stained cells $>50 \%$. Scores were obtained by increasing the strength and reactivity of the reaction. A score of 2 is defined as high expression, and a score below 2 is defined as low expression.

2.12. Western Blot. The primary antibodies used in this study against MCM2 (ab31159), MCM3 (ab128923), MCM4 (ab4459), MCM5 (ab75975), MCM6 (ab201683), MCM7 (ab52489), and GAPDH (ab181603) were obtained from Abcam (Cambridge, MA, USA). Western blotting was conducted according to our previous report [56].

2.13. Statistical Analysis. Statistical analyses were performed in the R Programming Language (version 3.6). All statistical tests were bilateral, and $P<0.05$ was statistically significant.

\section{Results}

3.1. The mRNA and Protein Expression of MCMs in HCC. Firstly, we utilized the Oncomine database to extract the data of MCM transcriptional levels in different cancer and corresponding normal tissues (Figure 1). The datasets of total unique analyses for MCM2, MCM3, MCM4, MCM5, MCM6, and MCM7 were 402, 446, 453, 436, 450, and 424, respectively. In cancer datasets, these MCMs were increased in most cancers, especially in bladder cancer, brain and CNS cancer, breast cancer, cervical cancer, colorectal cancer, esophageal cancer, gastric cancer, head and neck cancer, HCC, lung cancer, ovarian cancer, and sarcoma. Further- more, the level of MCM2 in cancer tissues was increased in 65 datasets and decreased in 2 datasets compared to normal tissues. The MCM3 level was significantly enhanced in 39 datasets but reduced in 2 datasets. For MCM4, 67 datasets indicated overexpression, but 9 datasets indicated low expression. The mRNA level of MCM5 was upregulated in 46 datasets but downregulated in 2 datasets. High expression of MCM6 was observed in 49 datasets, while low expression was detected in 4 datasets. Moreover, the increased level of MCM7 was found in 52 datasets, but decreased level was observed in 6 datasets.

MCM4 was also enhanced in HCC compared to normal tissues based on Wurmbach Liver datasets [3]. Chen Liver datasets showed an obviously increased MCM6 level in HCC [57]. In addition, Roessler Liver and Roessler Liver 2 datasets indicated that MCMs were both significantly increased in HCC compared to normal tissues [58]. The statistical significance results with corresponding $P$ values are shown in Figure 1 and Table 1.

We also used GEPIA to compare the transcriptional levels of MCMs in HCC and normal tissue (Figure 2). We found that the expression of both MCM proteins in tumor tissues was significantly upregulated. Moreover, the correlation between MCM level and HCC stages was also analyzed in GEPIA, which indicated that both MCMs were closely associated with HCC stage (Supplementary Figure S1).

In addition, the immunohistochemistry (IHC) staining images for MCM protein in HCC and normal liver tissues were extracted from the HPA database (Figure 3). We found that these proteins were both significantly increased in HCC tissues compared to normal liver tissues.

3.2. Prognostic Values of MCMs in HCC Patients. Then, we utilized the database of KM plotter to analyze the correlation with high expression of MCM2 and worse RFS (HR $=1.73$, $P=0.001)$. HCC patients with high level of MCM3 showed unfavorable RFS (HR $=1.81, P=0.00063)$. High expression of MCM4 had poor RFS (HR $=1.51, P=0.02)$. High levels of MCM5 were correlated with decreased RFS of HCC patients $(\mathrm{HR}=1.89, P=0.00018)$. Moreover, the level of MCM6 $(\mathrm{HR}=2.08, P=0.000012)$ and MCM7 $(\mathrm{HR}=1.78$, $P=0.0019)$ was also associated with RFS in HCC patients, respectively (Table 2 ).

The further analysis of these impacts on prognosis by these proteins is provided. We found that the prognosis significance of MCMs had a close correlation with some clinicopathological parameters, including clinical stages, pathology grade, and vascular invasion (Table 3). High expression of MCM2 was prominently associated with worse OS in HCC stage $1+2(\mathrm{HR}=2.13, P=0.0019)$ and stage $3+4(\mathrm{HR}=2.09$, $P=0.043)$. Likewise, similar results on stage $1+2$ and stage 3 +4 were also observed in $\mathrm{MCM} 3(\mathrm{HR}=1.85, P=0.0152$; $\mathrm{HR}=1.77, \quad P=0.0672), \quad \mathrm{MCM} 4 \quad(\mathrm{HR}=1.61, \quad P=0.0669$; $\mathrm{HR}=3.06, \quad P=0.000093), \quad$ MCM5 $\quad(\mathrm{HR}=1.92, P=0.009$; $\mathrm{HR}=1.84, \quad P=0.0357), \quad \mathrm{MCM} 6 \quad(\mathrm{HR}=2.34, \quad P=0.0007 ;$ $\mathrm{HR}=2.24, P=0.0081)$, and $\mathrm{MCM} 7(\mathrm{HR}=2.13, P=0.0019$; $\mathrm{HR}=2.09, P=0.043)$.

Both OS significance of MCMs had a significant correlation with no vascular invasion, which indicated that high 


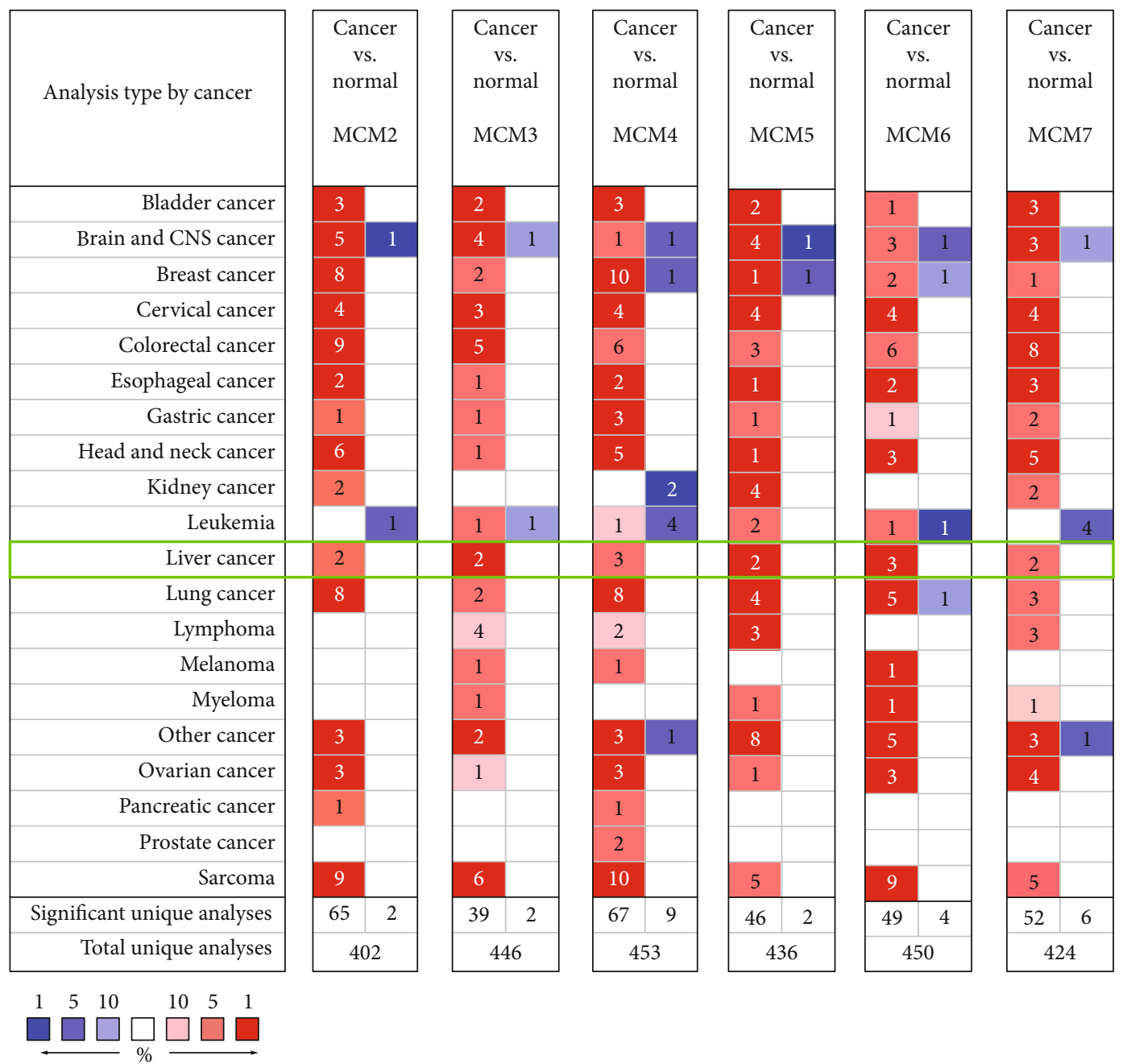

FIGURE 1: Oncomine analysis of MCMs in different cancer types. MCM expression between different cancer types and corresponding normal tissues was summarized. Threshold ( $P$ value $\leq 0.05$; $|\mathrm{FDR}| \geq 2$; gene rank $\leq 10 \%$; data type: $\mathrm{mRNA}$ ) is expressed in colored cells. In tumor tissue, red cells represent overexpression of the target gene compared to normal tissue, while blue cells represent downregulation of the gene. Gene levels are indicated by the color depth of the cell. (You can refer to the web version of this article to explain the color reference in the diagram.)

level of MCMs could predict the poor prognosis in HCC patients without vascular invasion. In the HCC patients with vascular invasion, only MCM7 could suggest a poor prognosis $(\mathrm{HR}=2.27, P=0.0328)$. Furthermore, the OS significance of MCM2 was not associated with grade $1(\mathrm{HR}=2.43, P=$ $0.0666)$, but was associated with grade $2(\mathrm{HR}=1.82, P=$ $0.0368)$ and grade $3(\mathrm{HR}=4.13, P=0.000088)$. Other MCMs are well summarized in Table 3 . Next, we analyzed the association between prognosis significance of MCMs and hepatitis virus infection and alcohol consumption, respectively. The results indicated that both MCM overexpression had a positive association with poor OS in HCC patients without hepatitis virus infection or alcohol consumption. Only MCM5 and MCM7 had a significant correlation with poor OS in HCC patients with alcohol consumption. MCM6 could predict the poor OS $(\mathrm{HR}=2.04, P=0.0316)$, RFS $(\mathrm{HR}=1.74$, $P=0.0258)$, PFS (HR $=1.7, P=0.0218)$, and DSS $(\mathrm{HR}=2.26$, $P=0.0469)$ in HCC patients with hepatitis virus infection. The detailed results are summarized in Table 4.
3.3. MCM Genetic Alteration in HCC Patients. By using the cBioPortal database, we found that the percentages of MCM genetic alterations were $15.92 \%, 5.63 \%$, and $2.88 \%$ in three datasets, including INSERM, AMC, and TCGA (Figure 4(a)). However, we analyzed the correlation between MCM gene alterations and survival outcome, which indicated that cases of MCM gene modification were not associated with OS $(P=0.0957$, Figure $4(\mathrm{~b}))$. The alteration frequency of MCM2, MCM3, MCM4, MCM5, MCM6, and MCM7 was $1.1 \%, 2.7 \%, 3 \%, 0.9 \%, 1 \%$, and $1.7 \%$ based on six datasets, including MSK, INSERM, MSK, AMC, RIKEN, and TCGA (Figure 4(c)).

3.4. Correlation Analyses of MCMs in HCC Patients. We extracted the MCM mRNA level between each other in HCC from TCGA Provisional dataset (RNA Seq V2 RSEM) by using the cBioPortal. Spearman's correlation analysis among these MCM levels suggested significantly positive correlation between both MCM2/3/4/5/6/7 and other MCMs (Figure 5(a)). 
TABLE 1: Differential expression analyses of MCMs in HCC.

\begin{tabular}{lccccccc}
\hline Gene & Database & Normal (cases) & Cancer (cases) & Fold change & $t$-test & $P$ value & Reference \\
\hline \multirow{2}{*}{ MCM2 } & Roessler Liver & Normal liver (21) & Hepatocellular carcinoma [22] & 3.252 & 7.289 & $3 E-08$ & {$[57]$} \\
& Roessler Liver 2 & Normal liver (220) & Hepatocellular carcinoma (225) & 3.144 & 21.853 & $2.7 E-64$ & {$[57]$} \\
\multirow{2}{*}{ MCM3 } & Roessler Liver & Normal liver (21) & Hepatocellular carcinoma [22] & 2.953 & 7.55 & $1.5 E-08$ & {$[57]$} \\
& Roessler Liver 2 & Normal liver (220) & Hepatocellular carcinoma (225) & 3.023 & 23.777 & $5.7 E-72$ & {$[57]$} \\
& Wurmbach Liver & Normal liver [10] & Hepatocellular carcinoma [35] & 2.67 & 5.936 & $2.5 E-07$ & {$[3]$} \\
MCM4 & Roessler Liver & Normal liver [21] & Hepatocellular carcinoma [22] & 2.541 & 6.958 & $6 E-08$ & {$[57]$} \\
& Roessler Liver 2 & Normal liver (220) & Hepatocellular carcinoma (225) & 3.044 & 22.497 & $5.3 E-66$ & {$[57]$} \\
\multirow{2}{*}{ MCM5 } & Roessler Liver & Normal liver [21] & Hepatocellular carcinoma [22] & 3.353 & 8.167 & $3.9 E-10$ & {$[57]$} \\
& Roessler Liver 2 & Normal liver (220) & Hepatocellular carcinoma (225) & 2.752 & 20.76 & $8.8 E-64$ & {$[57]$} \\
\multirow{2}{*}{ MCM6 } & Roessler Liver & Normal liver [21] & Hepatocellular carcinoma [22] & 3.353 & 8.167 & $3.9 E-10$ & {$[57]$} \\
& Roessler Liver 2 & Normal liver (220) & Hepatocellular carcinoma (225) & 2.752 & 20.76 & $8.8 E-64$ & {$[57]$} \\
& Chen Liver & Normal liver (76) & Hepatocellular carcinoma (104) & 2.023 & 7.755 & $4 E-13$ & {$[56]$} \\
MCM7 & Roessler Liver & Normal liver [21] & Hepatocellular carcinoma [22] & 2.453 & 7.019 & $2.7 E-08$ & {$[57]$} \\
& Roessler Liver 2 & Normal liver (220) & Hepatocellular carcinoma (225) & 2.154 & 19.734 & $1.6 E-57$ & {$[57]$} \\
\hline
\end{tabular}

$P$ values $\leq 0.05$ were considered statistically significant.

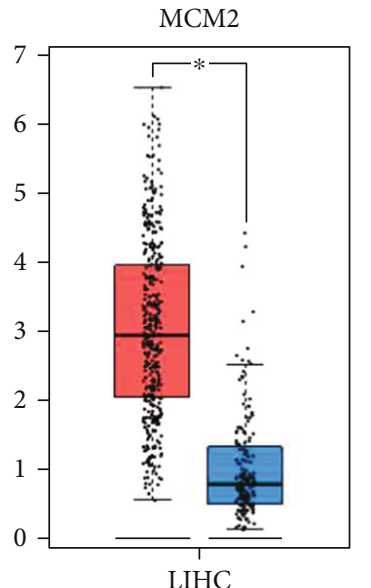

$(\operatorname{num}(t)=369 ; \operatorname{num}(n)=160)$

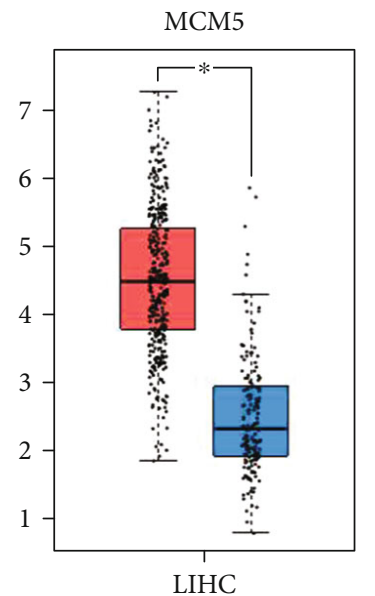

$(\operatorname{num}(t)=369 ; \operatorname{num}(n)=160)$

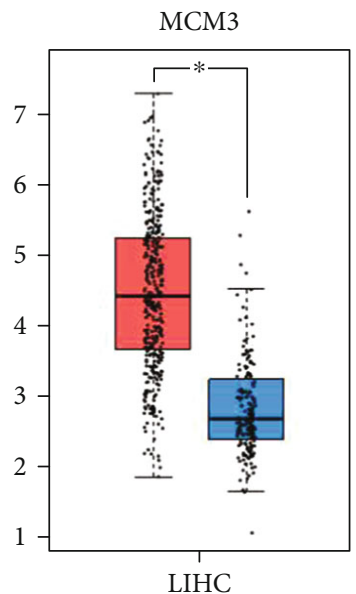

$(\operatorname{num}(t)=369 ; \operatorname{num}(n)=160)$

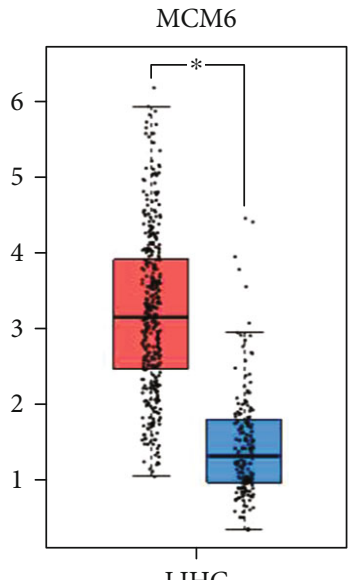

$(\operatorname{num}(t)=369 ; \operatorname{num}(n)=160)$

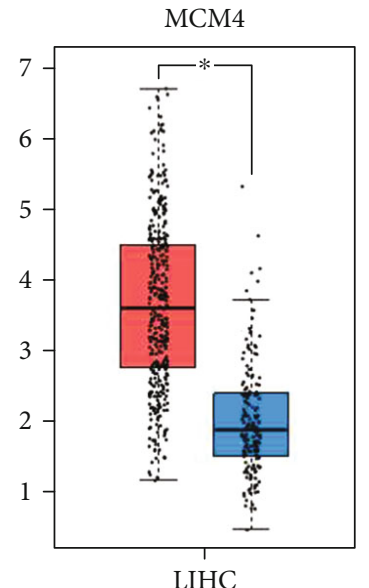

$(\operatorname{num}(t)=369 ; \operatorname{num}(n)=160)$

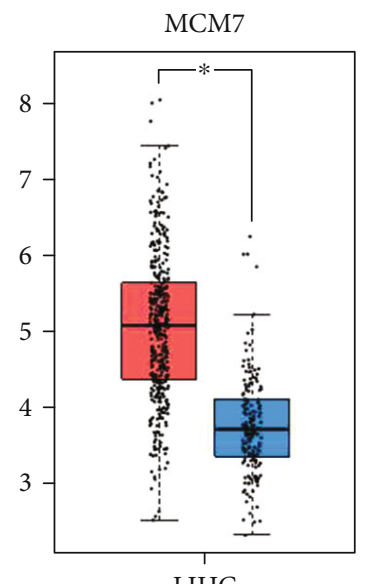

$(\operatorname{num}(t)=369 ; \operatorname{num}(n)=160)$

Cancer

Normal

Figure 2: The mRNA expression levels of MCMs by GEPIA analysis in HCC. Box plots of individual MCM level in HCC tissues and normal liver tissues, $P$ value $\leq 0.05$. 


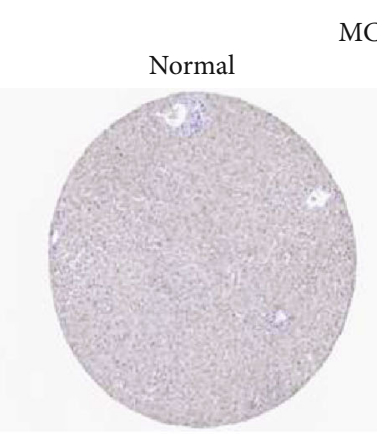

Staining: no detected

Antibody HPA031495

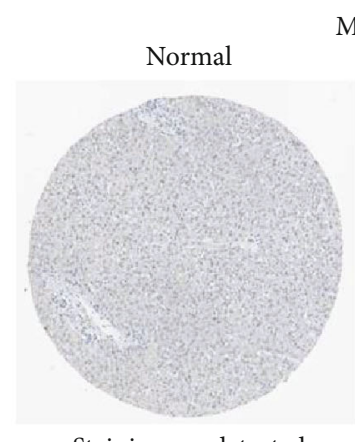

Staining: no detected

MCM4

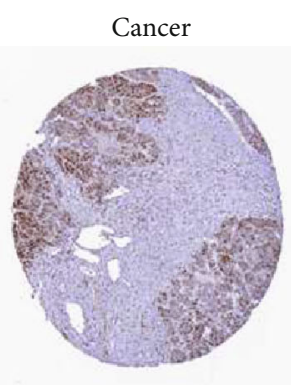

Staining: strong

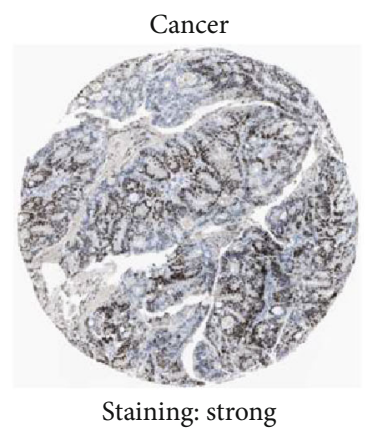

Antibody HPA004873

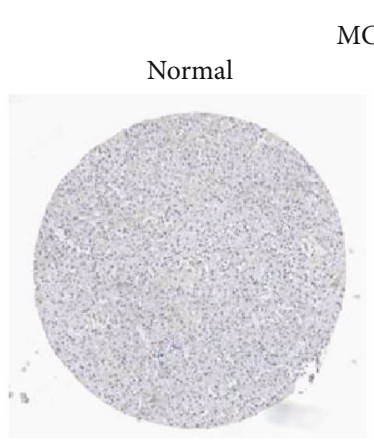

Staining: no detected

MCM6

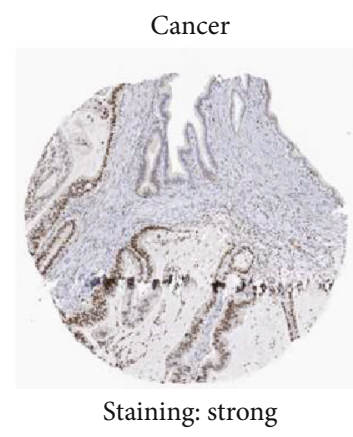

Antibody HPA004818

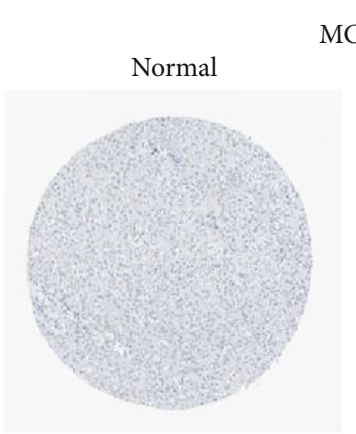

Staining: no detected

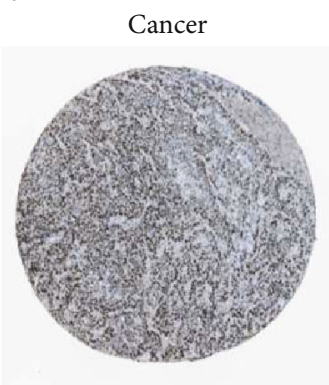

Staining: strong

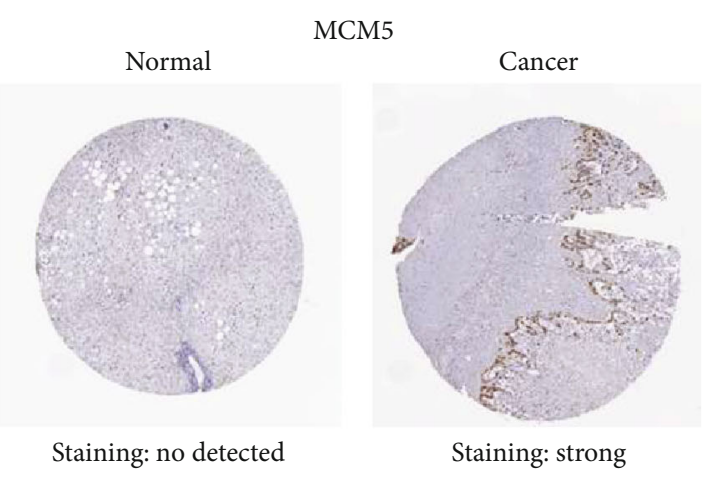

Antibody HPA000845

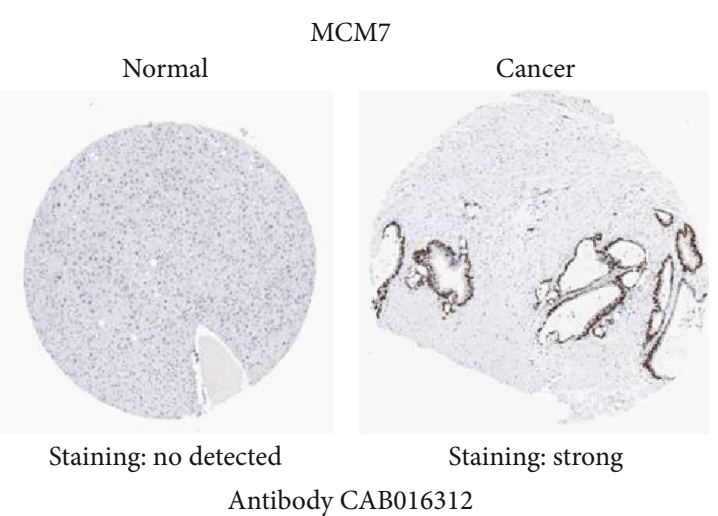

Figure 3: The protein expression of MCMs in HCC shown by immunohistochemistry staining images based on the Human Protein Atlas.

Next, we utilized the GeneMANIA tools to analyze the association of MCMs at gene level (Figure 5(b)). This result indicated that the physical interactions among MCM2-7 were significant in this network, which might attribute to the shared protein domains. Relationships were significantly found among MCM2-7 in coexpression. Furthermore, pathway was noticed in reactome among MCM2-7 and other key genes, including CDC45, CDC7, ORC6, MCM10, CDT1, ORC5, GINS4, CLSPN, ORC4, and POLD3.

We further identified the protein interactions of MCM27 by the STRING database (Figure 5(c)). The interactions among MCM2-7 were shown in experiments, databases, and coexpression. Moreover, the network for MCM2-7 and the 40 altered neighboring genes was constructed, such as
MCMBP, GINS3, GINS2, GINS1, POLA2, CDC7, DBF4, PRIM1, CDC6, ORC3, LRWD1, ORC4, ORC5, CDC45, TIPIN, POLE2, RFC3, ORC6, ORC2, ORC1, GMNN, CCNA2, CDT1, MCM8, MCM10, POLA1, RPA2, RFC4, TIMELESS, RAD52, RPA1, RPA3, GINS4, CDK2, CLSPN, CHEK1, BLM, WRN, RMI1, and TOP3A. The detailed results are shown in Figure 6(c).

3.5. Functional Enrichment Analysis of MCMs in HCC. Finally, we excavated GO and KEGG pathway data for MCMs and their 40 altered neighboring genes by using Metascape. Top 5 KEGG pathways were DNA replication, cell cycle, homologous recombination, pyrimidine metabolism, and viral carcinogenesis (Figures 6(a) and 6(b)). Top 
TABLE 2: KM plotter showing the correlation between different MCMs and survival outcomes in hepatic carcinoma.

\begin{tabular}{|c|c|c|c|c|c|c|c|c|}
\hline Gene & RNAseq ID & Survival outcome & No. of cases & HR & $95 \% \mathrm{CI}$ & $P$ value & $\begin{array}{c}\text { Low-expression cohort } \\
\text { (months) }\end{array}$ & $\begin{array}{l}\text { High-expression cohort } \\
\text { (months) }\end{array}$ \\
\hline \multirow{4}{*}{ MCM2 } & \multirow{4}{*}{4171} & OS & 364 & 1.96 & $1.38-2.7$ & 0.0001 & 71 & 38.3 \\
\hline & & RFS & 313 & 1.73 & $1.24-2.4$ & 0.001 & 36.1 & 13.27 \\
\hline & & PFS & 366 & 1.87 & $1.34-2.6$ & 0.0002 & 40.97 & 15.83 \\
\hline & & DSS & 357 & 2.34 & $1.49-3.6$ & 0.00015 & 84.4 & 61.73 \\
\hline \multirow{4}{*}{ MCM3 } & \multirow{4}{*}{4172} & Os & 364 & 1.8 & $1.25-2.5$ & 0.0013 & 71 & 46.6 \\
\hline & & RFS & 313 & 1.81 & $1.28-2.5$ & 0.00063 & 40.97 & 15.97 \\
\hline & & PFS & 366 & 1.86 & $1.36-2.5$ & 0.000077 & 36.27 & 13.33 \\
\hline & & DSS & 357 & 2.47 & $1.5-4.06$ & 0.00025 & 104.17 & 81.87 \\
\hline \multirow{4}{*}{ MCM4 } & \multirow{4}{*}{4173} & Os & 364 & 1.9 & $1.31-2.7$ & 0.00058 & 70.5 & 25.6 \\
\hline & & RFS & 313 & 1.51 & $1.06-2.1$ & 0.02 & 34.4 & 13.27 \\
\hline & & PFS & 366 & 1.53 & $1.12-2.0$ & 0.0068 & 29.3 & 12.87 \\
\hline & & DSS & 357 & 2.27 & $1.42-3.6$ & 0.00043 & 84.4 & 49.67 \\
\hline \multirow{4}{*}{ MCM5 } & \multirow{4}{*}{4174} & Os & 364 & 1.94 & $1.36-2.7$ & 0.00019 & 70.5 & 30 \\
\hline & & RFS & 313 & 1.89 & $1.35-2.6$ & 0.00018 & 37.23 & 12.87 \\
\hline & & PFS & 366 & 1.79 & $1.32-2.4$ & 0.00014 & 29.77 & 11.47 \\
\hline & & DSS & 357 & 2.24 & $1.43-3.5$ & 0.00031 & 84.4 & 56.17 \\
\hline \multirow{4}{*}{ MCM6 } & \multirow{4}{*}{4175} & OS & 364 & 2.29 & $1.61-3.2$ & 0.0000023 & 70.5 & 24.1 \\
\hline & & RFS & 313 & 2.08 & $1.49-2.9$ & 0.000012 & 42.630 & 13.33 \\
\hline & & PFS & 366 & 2.11 & $1.56-2.8$ & 0.00000059 & 36.27 & 11.97 \\
\hline & & DSS & 357 & 2.73 & $1.74-4.2$ & 0.0000056 & 84.4 & 49.67 \\
\hline \multirow{4}{*}{ MCM7 } & \multirow{4}{*}{4176} & OS & 364 & 1.93 & $1.36-2.7$ & 0.00019 & 71 & 38.3 \\
\hline & & RFS & 313 & 1.78 & $1.23-2.5$ & 0.0019 & 42.87 & 18.3 \\
\hline & & PFS & 366 & 1.86 & $1.33-2.6$ & 0.00022 & 36.27 & 15.17 \\
\hline & & DSS & 357 & 2.64 & $1.52-4.5$ & 0.00035 & 59.7 & 24.13 \\
\hline
\end{tabular}

$P$ values $\leq 0.05$ were considered statistically significant.

20 GO enrichment are shown in Figures 6(c) and 6(d). Biological processes are as follows: DNA replication, DNAdependent DNA replication, DNA replication initiation, nuclear DNA replication, double-strand break repair via homologous recombination, DNA strand elongation involved in DNA replication, telomere maintenance via semiconservative replication, regulation of DNA replication, DNA replication checkpoint, DNA replication preinitiation complex assembly, negative regulation of DNA replication, protein localization to chromosome, chromosome separation, G2 DNA damage checkpoint, and histone phosphorylation; cellular components are as follows: replication fork, chromosome, telomeric region, replication fork protection complex, chromatin, and centrosome. Next, the proteinprotein interaction enrichment analysis revealed that biological functions were mostly connected with the activation of ATR in response to replication stress, activation of the prereplicative complex, DNA replication preinitiation, processing of DNA double-strand break ends, HDR through homologous recombination (HRR) or single-strand annealing (SSA), and homology directed repair (Figures 6(e) and 6(f)).

3.6. Expressions of MCM2-7 Were Increased in HCC. To further demonstrate the level of MCM2-7 in HCC, we detected the levels of MCM2-7 in several HCC cell lines and normal hepatocyte cell lines. The results indicated that the levels of MCM2-7 were increased in HCC cell lines (HepG2, SNU368, SNU-354, HLE, and HLF) compared to the normal hepatocyte cell line (HL-7702). As shown in Figures 7(a) and 7(b), the expression of MCM2-7 in HCC cell lines significantly enhanced in the mRNA and protein level, which was consistent with the database analysis. Furthermore, the correlation analysis among these MCMs also suggested the significant positive correlation between these MCMs in HCC cell lines and normal hepatocyte cell line in the protein level (Figure 7(a) and Supplementary Figure S2). The next section of the survey was concerned with MCM expression in HCC tissues. The results, as shown in Figure $7(\mathrm{c})$, indicated that MCMs were significantly enhanced in HCC tissues compared to paracarcinoma tissues. Together, these results provided important insights into the fact that MCMs might play significant roles in the formation, development, and progression of HCC.

\section{Discussion}

Currently, more and more studies indicated that ectopic expression of MCMs could promote DNA replication [12] and accelerate cell cycle [11] and metastasis [14]. MCMs were involved in the development and progression of many 
TABLE 3: The correlation between MCMs and survival outcomes in pathology parameters of hepatic carcinoma.

\begin{tabular}{|c|c|c|c|c|c|c|c|c|c|c|c|c|c|c|c|c|}
\hline \multirow{2}{*}{ Gene } & \multirow{2}{*}{$\begin{array}{l}\text { Survival } \\
\text { outcome }\end{array}$} & \multicolumn{2}{|c|}{ Stage $1+2$} & \multicolumn{2}{|c|}{ Stage $3+4$} & \multicolumn{2}{|c|}{ Grade 1} & \multicolumn{2}{|c|}{ Grade 2} & \multicolumn{2}{|c|}{ Grade 3} & Grade 4 & \multicolumn{2}{|c|}{$\begin{array}{l}\text { Vascular } \\
\text { invasion - }\end{array}$} & \multicolumn{2}{|c|}{$\begin{array}{c}\text { Vascular } \\
\text { invasion }+\end{array}$} \\
\hline & & HR & $P$ value & HR & $P$ value & HR & $P$ value & HR & $P$ value & HR & $P$ value & HR $P$ value & HR & $P$ value & $\mathrm{HR}$ & $P$ value \\
\hline \multirow{4}{*}{ MCM2 } & OS & 2.13 & 0.0019 & 2.09 & 0.043 & 2.43 & 0.0666 & 1.82 & 0.0368 & 4.13 & $9 E-05$ & & 1.99 & 0.0113 & 2.21 & 0.0789 \\
\hline & RFS & 1.66 & 0.0281 & 2.06 & 0.0311 & 1.68 & 0.288 & 2.65 & $6 E-05$ & 1.58 & $\begin{array}{c}1.58 \\
0.1737\end{array}$ & & 1.72 & 0.0261 & 1.72 & 0.0922 \\
\hline & PFS & 1.75 & 0.0082 & 2.06 & 0.02 & 0.02 & 0.0534 & 2.38 & $6 E-05$ & 1.39 & 0.2373 & & 1.91 & 0.0101 & 1.43 & 0.2257 \\
\hline & DSS & 5.96 & 0.0002 & 2.31 & 0.058 & 2.05 & 0.2312 & 3.9 & 0.0012 & 4.62 & 0.0007 & & 2.79 & 0.0088 & 1.43 & 0.5532 \\
\hline \multirow{4}{*}{ MCM3 } & OS & 1.85 & 0.0152 & 1.77 & 0.0672 & 3.14 & 0.0164 & 1.73 & 0.0373 & 1.6 & 0.1314 & & 1.94 & 0.0147 & 0.77 & 0.5264 \\
\hline & RFS & 1.66 & 0.0174 & 1.38 & 0.3721 & 3.6 & 0.0164 & 2.01 & 0.0049 & 2.06 & 0.0435 & & 1.85 & 0.0138 & 1.74 & 0.0919 \\
\hline & PFS & 1.75 & 0.0046 & 1.39 & 0.2206 & 2.91 & 0.0096 & 2.12 & 0.0006 & 1.79 & 0.0759 & & 1.97 & 0.005 & 1.77 & 0.0547 \\
\hline & DSS & 2.7 & 0.0079 & 1.78 & 0.1291 & 4.81 & 0.0084 & 2.28 & 0.016 & 2.02 & 0.0728 & & 2.34 & 0.0264 & 2.43 & 0.166 \\
\hline \multirow{4}{*}{ MCM4 } & OS & 1.61 & 0.0669 & 3.06 & 3.06 & 2.18 & 0.1172 & 1.72 & 0.0662 & 2.68 & 0.0008 & & 1.82 & 0.0287 & 1.6 & 0.244 \\
\hline & RFS & 1.23 & 0.382 & 2.21 & 0.0115 & 0.67 & 0.4853 & 1.67 & 0.0596 & 1.81 & 0.0346 & & 1.74 & 0.0586 & 0.57 & 0.098 \\
\hline & PFS & 1.34 & 0.1646 & 1.91 & 0.0168 & 1.58 & 0.2514 & 1.83 & 0.0112 & 1.62 & 0.0549 & & 1.58 & 0.0521 & 0.68 & 0.2275 \\
\hline & DSS & 1.88 & 0.0987 & 3.26 & 0.0006 & 4.53 & 0.018 & 2.34 & 0.0164 & 4.13 & 0.0002 & & 2.18 & 2.18 & 0.6 & 0.392 \\
\hline \multirow{4}{*}{ MCM5 } & OS & 1.92 & 0.009 & 1.84 & 0.0357 & 3.34 & 0.0293 & 1.69 & 0.0422 & 2.03 & 0.0189 & & 1.93 & 0.0124 & 0.63 & 0.2394 \\
\hline & RFS & 1.65 & 0.0192 & 1.84 & 0.0486 & 1.49 & 0.4876 & 2.53 & 0.0001 & 1.59 & 0.0872 & & 1.74 & 0.0243 & 2.47 & 0.0051 \\
\hline & PFS & 1.64 & 0.016 & 2.02 & 0.0129 & 2.14 & 0.0539 & 2.27 & 0.0002 & 1.49 & 0.1165 & & 1.63 & 0.032 & 1.9 & 0.0276 \\
\hline & DSS & 2.26 & 0.0205 & 2.6 & 0.0094 & 7.89 & 0.0052 & 1.58 & 0.1959 & 2.83 & 0.0068 & & 2.01 & 0.0554 & 0.42 & 0.1087 \\
\hline \multirow{4}{*}{ MCM6 } & OS & 2.34 & 0.0007 & 2.24 & 0.0081 & 2.72 & 0.0325 & 2.8 & 0.0001 & 2.71 & 0.008 & & 2.07 & 0.0091 & 1.75 & 0.1493 \\
\hline & RFS & 1.79 & 0.0056 & 2.66 & 0.0044 & 1.81 & 0.2221 & 2.56 & 0.0001 & 2.42 & 0.0043 & & 2.06 & 0.0028 & 0.66 & 0.2216 \\
\hline & PFS & 1.94 & 0.0005 & 2.12 & 0.0098 & 2.56 & 0.0184 & 2.62 & $8 E-06$ & 2.41 & 0.0029 & & 1.97 & 0.0023 & 1.45 & 0.2057 \\
\hline & DSS & 2.96 & 0.0017 & 2.34 & 0.0129 & 2.81 & 0.0861 & 3.95 & $3 E-05$ & 3.15 & 0.003 & & 3 & 0.0209 & 0.56 & 0.2984 \\
\hline \multirow{4}{*}{ MCM7 } & OS & 2.13 & 0.0019 & 2.09 & 0.043 & 2.43 & 0.0666 & 1.82 & 0.0368 & 4.13 & $9 E-05$ & & 1.91 & 0.012 & 2.27 & 0.0328 \\
\hline & RFS & 1.66 & 0.0281 & 2.06 & 0.0311 & 1.68 & 0.288 & 2.65 & $6 E-05$ & 1.58 & 0.1737 & & 1.57 & 0.0913 & 1.55 & 0.171 \\
\hline & PFS & 1.75 & 0.0082 & 2.06 & 0.02 & 2.12 & 0.0534 & 2.38 & $6 E-05$ & 1.39 & 0.2373 & & 1.4 & 0.1539 & 1.76 & 0.0548 \\
\hline & DSS & 5.96 & 0.0002 & 2.31 & 0.0587 & 2.05 & 0.2312 & 3.9 & 0.0012 & 4.62 & 0.0007 & & 2.01 & 0.0853 & 1.9 & 0.2445 \\
\hline
\end{tabular}

$P$ values $\leq 0.05$ were considered statistically significant.

human diseases [59]. In previous studies, the heterohexameric complex composed of MCM2-7 has been well summarized in human cancer cells. However, there are still many questions to be systematically solved about the expression, function, interaction, and prognostic value of MCMs in HCC. Therefore, we conducted a comprehensive analysis to reveal the transcriptional level, function enrichment, gene/protein interaction, and prognostic values of MCM2-7 in HCC.

In this study, we found that the expression of MCM2-7 was significantly increased in HCC tissues compared to normal liver tissues. MCM2, an oncogene, was correlated with the development and progression from cirrhosis to HCC [60]. MCM2 protein strongly expressed in high-grade squamous intraepithelial lesion may be useful as a cascade screening tool for detecting precancerous changes in cervical cancer [61]. Our results indicated that high level of MCM2 was significantly associated with worse OS/RFS/PFS/DSS in HCC patients without hepatitis virus infection. Zhao et al. revealed that MCM3 was a better marker of proliferation than Ki67, making it a valuable prognostic tool independent of ER and HER2 states [62]. In the group of nonalcohol consumption, our study indicated that HCC patients with elevated MCM3 expression had a bad OS/RFS/PFS/DSS. Choy et al. indicated that MCM4 could be used as a more sensitive proliferative marker for the identification of esophageal lesions [63]. We found that HCC patients with elevated MCM4 mRNA levels had unfavorable RFS and OS. Gong and his colleagues revealed that ectopic expression of MCM5 had a close correlation with malignancy and poor prognosis, which might be a potential prognostic marker in renal cell carcinoma [64]. In the study, we found that high level of MCM5 had also close correlation with the poor prognosis of HCC, especially in OS/RFS/PFS/DSS. Liu et al. suggested that MCM6 could indicate poor prognosis and promote migration and invasion, which could be predicted preclinical early recurrence in HCC patients to indicate more careful monitoring and aggressive treatment intervention [14]. Similarly, we also found MCM6 expression predicted poor OS and PFS in stage $1+2$, stage $3+4$, grade $1 / 2 / 3$, or nonvascular invasion patients. Furthermore, MCM7 has advantages over traditional cell cycle markers, such as Ki67 and PCNA, because it has a higher sensitivity and is less susceptible to external factors, including inflammatory factors [65]. Given that Ki67 and PCNA expression can only be observed at certain stages of replication and can be easily interfered with, the presence 
TABLE 4: The correlation between MCMs and survival outcomes in hepatic carcinoma based upon the alcohol consumption and hepatitis virus status.

\begin{tabular}{|c|c|c|c|c|c|c|c|c|c|}
\hline \multirow{2}{*}{ Gene } & \multirow[t]{2}{*}{ Survival outcome } & \multicolumn{2}{|c|}{$\begin{array}{c}\text { Alcohol } \\
\text { consumption - }\end{array}$} & \multicolumn{2}{|c|}{$\begin{array}{c}\text { Alcohol } \\
\text { consumption + }\end{array}$} & \multicolumn{2}{|c|}{ Hepatitis virus - } & \multicolumn{2}{|c|}{ Hepatitis virus + } \\
\hline & & HR & $P$ value & HR & $P$ value & HR & $P$ value & HR & $P$ value \\
\hline \multirow{4}{*}{ MCM2 } & OS & 2.43 & 0.00022 & 2.09 & 0.0578 & 3.37 & 0.00001 & 1.73 & 0.1849 \\
\hline & RFS & 2 & 0.0018 & 2.25 & 0.012 & 3.17 & 0.0000054 & 1.33 & 0.2661 \\
\hline & PFS & 1.85 & 0.0024 & 2.37 & 0.0031 & 2.67 & 0.000022 & 1.45 & 0.1802 \\
\hline & DSS & 3.4 & 0.000043 & 1.77 & 0.1096 & 3.25 & 0.000032 & 2.55 & 0.0326 \\
\hline \multirow{4}{*}{ MCM3 } & OS & 1.87 & 0.0153 & 1.77 & 0.0696 & 2.69 & 0.000055 & 1.52 & 0.2548 \\
\hline & RFS & 2.1 & 0.0012 & 2.18 & 0.0447 & 2.37 & 0.0007 & 1.85 & 0.0272 \\
\hline & PFS & 2.28 & 0.0004 & 1.77 & 0.0412 & 2.53 & 0.000024 & 1.8 & 0.028 \\
\hline & DSS & 3.66 & 0.0009 & 1.78 & 0.1115 & 4.07 & 0.000011 & 2.76 & 0.0547 \\
\hline \multirow{4}{*}{ MCM4 } & OS & 2.34 & 0.0004 & 1.51 & 0.203 & 3.09 & 0.0000021 & 1.52 & 0.2329 \\
\hline & RFS & 1.3 & 0.2924 & 2.25 & 0.014 & 3.32 & 0.0000053 & 0.64 & 0.1563 \\
\hline & PFS & 1.43 & 0.1033 & 1.87 & 0.0168 & 3.12 & 0.00000047 & 0.75 & 0.2438 \\
\hline & DSS & 3.22 & 0.0002 & 2.43 & 0.0126 & 5.01 & 0.000000011 & 2.9 & 0.0721 \\
\hline \multirow{4}{*}{ MCM5 } & Os & 1.74 & 0.0208 & 2.59 & 0.0028 & 2.3 & 0.0004 & 1.87 & 0.0582 \\
\hline & RFS & 1.9 & 0.0047 & 2.48 & 0.0024 & 2.94 & 0.000017 & 1.32 & 0.2703 \\
\hline & PFS & 1.74 & 0.0078 & 2.22 & 0.002 & 2.57 & 0.000018 & 1.3 & 0.2851 \\
\hline & DSS & 2.56 & 0.0019 & 2.78 & 0.005 & 2.79 & 0.0002 & 2.32 & 0.0392 \\
\hline \multirow{4}{*}{ MCM6 } & OS & 2.7 & 0.000019 & 1.66 & 0.1114 & 2.86 & 0.0000058 & 2.04 & 0.0316 \\
\hline & RFS & 2.37 & 0.0002 & 2.24 & 0.0074 & 3.37 & 0.0000019 & 1.74 & 0.0258 \\
\hline & PFS & 2.44 & 0.00002 & 2.41 & 0.001 & 3.48 & 0.000000019 & 1.7 & 0.0218 \\
\hline & DSS & 4.28 & 0.00000063 & 1.93 & 0.067 & 4.5 & 0.00000013 & 2.26 & 0.0469 \\
\hline \multirow{4}{*}{ MCM7 } & OS & 2.13 & 0.0019 & 2.56 & 0.029 & 3.07 & 0.0627 & 1.75 & 0.0889 \\
\hline & RFS & 1.92 & 0.0035 & 3.21 & 0.0059 & 3.02 & 0.000038 & 1.41 & 0.2594 \\
\hline & PFS & 1.95 & 0.0035 & 3.06 & 0.0014 & 2.99 & 0.0000035 & 1.59 & 0.1061 \\
\hline & DSS & 3.62 & 0.0003 & 2.44 & 0.0627 & 3.95 & 0.00001 & 1.93 & 0.2238 \\
\hline
\end{tabular}

$P$ values $\leq 0.05$ were considered statistically significant.

of MCMs at all cellular stages may be the reason for the advantage [66]. Likewise, we analyzed the correlation between MCM7 level and prognosis, which indicated that MCM7could also be a valuable prognostic marker for HCC patients.

The relationship between MCMs and genetic alteration was observed in HCC using the cBioPortal database. However, the HCC prognosis was independent of the mutation, which might indicate that ectopic expression of MCMs was induced by other ways in HCC, such as protein phosphorylation, slunoylation, and ubiquitination. The epigenetic modifications, such as DNA methylation, histone acetylation, and noncoding RNA regulation, were still unclear in the MCM complex. HCC, as an acquired disease, may be more due to the epigenetic modifications and abnormal molecular signal transduction than to genetic factor and gene alteration $[4,5]$. Therefore, further analyses of protein and epigenetic modification are needed for the comparison with the analyses of gene alteration. The mechanism of gene alteration of these MCMs also needed further exploration.

Furthermore, our results suggested that there was a significant positive correlation between MCM proteins which were both increased in HCC compared to normal liver tis- sues. GeneMANIA analysis revealed that MCM2-7 had a close association with CDC45, CDC7, ORC6, MCM10, CDT1, ORC5, GINS4, CLSPN, ORC4, and POLD3 at the gene level. These interactions were involved in reactome. STRING analysis also indicated that MCM2-7 had a significant correlation with MCMBP, GINS3, GINS2, GINS1, POLA2, CDC7, DBF4, PRIM1, CDC6, ORC3, LRWD1, ORC4, ORC5, CDC45, TIPIN, POLE2, RFC3, ORC6, ORC2, ORC1, GMNN, CCNA2, CDT1, MCM8, MCM10, POLA1, RPA2, RFC4, TIMELESS, RAD52, RPA1, RPA3, GINS4, CDK2, CLSPN, CHEK1, BLM, WRN, RMI1, and TOP3A, which forms an important network to perform a series of pathophysiological functions at the protein level. Wen et al. constructed a network in association with small cell lung cancer by bioinformatics analysis, indicating that the interactions among MCM2/3/6 and other hub protein were involved in carcinogenesis [67].

In order to further explore the related functions and signaling pathways of these proteins, we studied the functional enrichment of MCMs and its mechanism by Metascape. The results indicated that the pathways involved in MCMs might contain DNA replication, cell cycle, homologous recombination, pyrimidine metabolism, and viral 


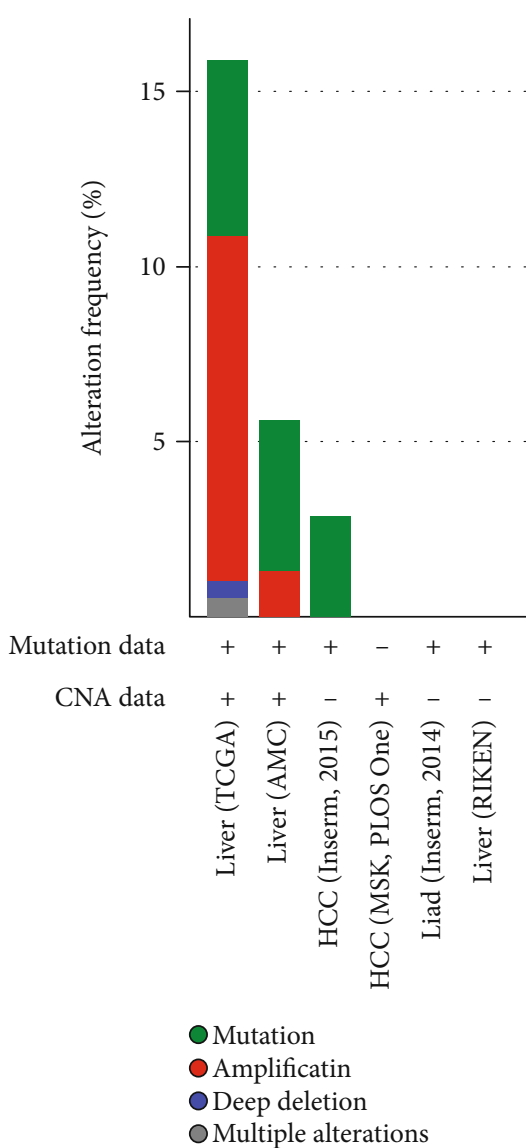

(a)

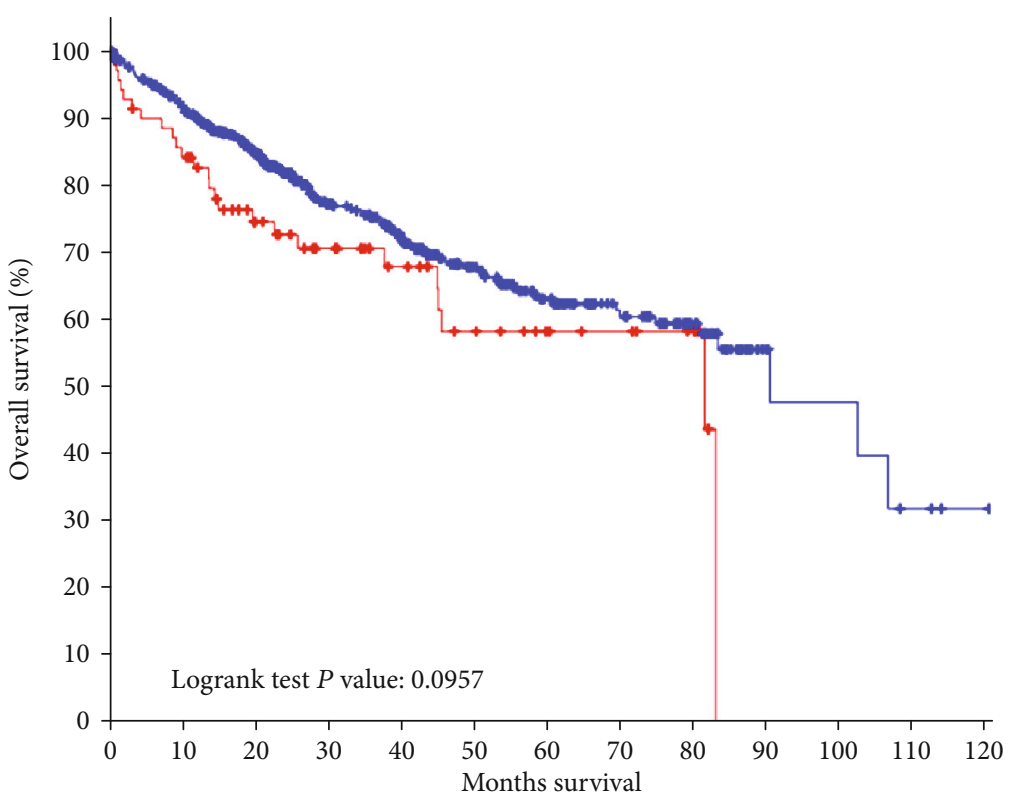

Overall survival Kalpan-Meier estimate

- Altered group

Unaltered group

(b)

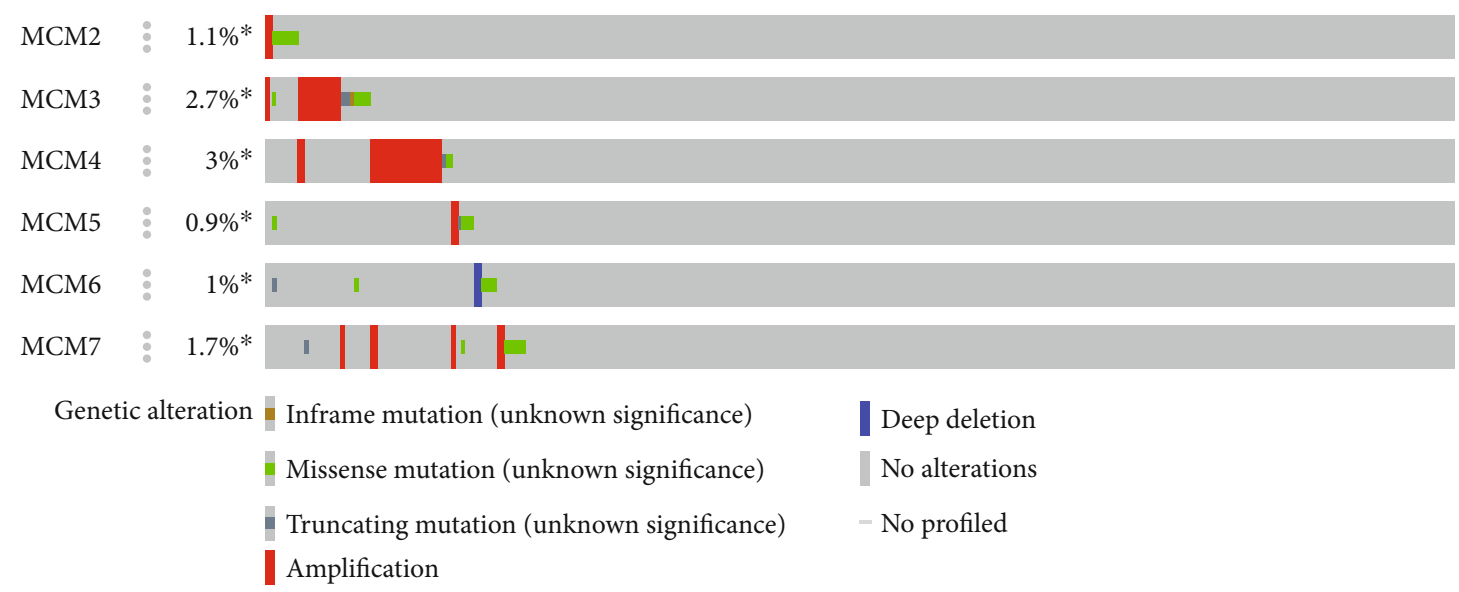

(c)

Figure 4: MCM alteration in HCC (cBioPortal). (a) MCM genetic alteration in TCGA firehose legacy datasets, AMC hepatology 2014 datasets, and INSERM Nat Genet 2015 datasets. (b) Kaplan-Meier plots comparing OS in HCC patients with or without MCM genetic alterations. (c) Alteration frequency of MCMs based on the cBioPortal dataset.

carcinogenesis. These pathways were frequent disorder in carcinogenesis. For example, Lin and his colleagues found that DNA replication could accelerate the cell cycle to promote carcinogenesis by the MCM complex [68]. Breast cancer type 1 susceptibility protein (BRCA1), a tumor suppressor, induces DNA double-strand break repair by homologous recombination, protecting DNA replication forks from attrition [69]. Sweeney et al. found that the combination of glutamine and glutamine-derived metabolites in purine and pyrimidine synthesis was inhibited by dimethylaminopurine and rhodoxin, effectively blocking the key biosynthetic pathway for the survival of leukemia cells [70]. Viral carcinogenesis has been demonstrated in nasopharyngeal carcinoma [71] and HCC [72] by EBV and hepatitis virus infection, 


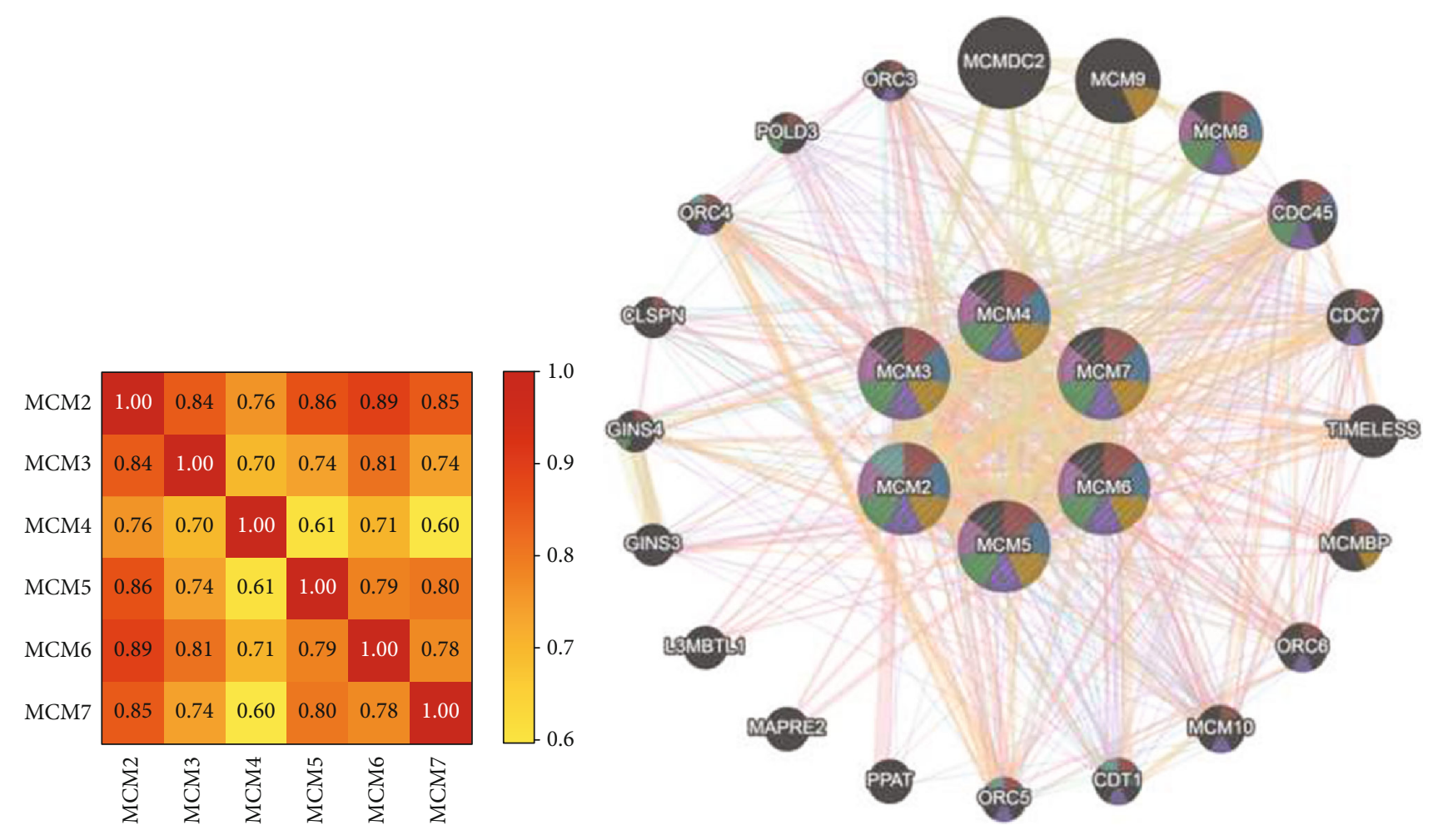

(a)

(b)

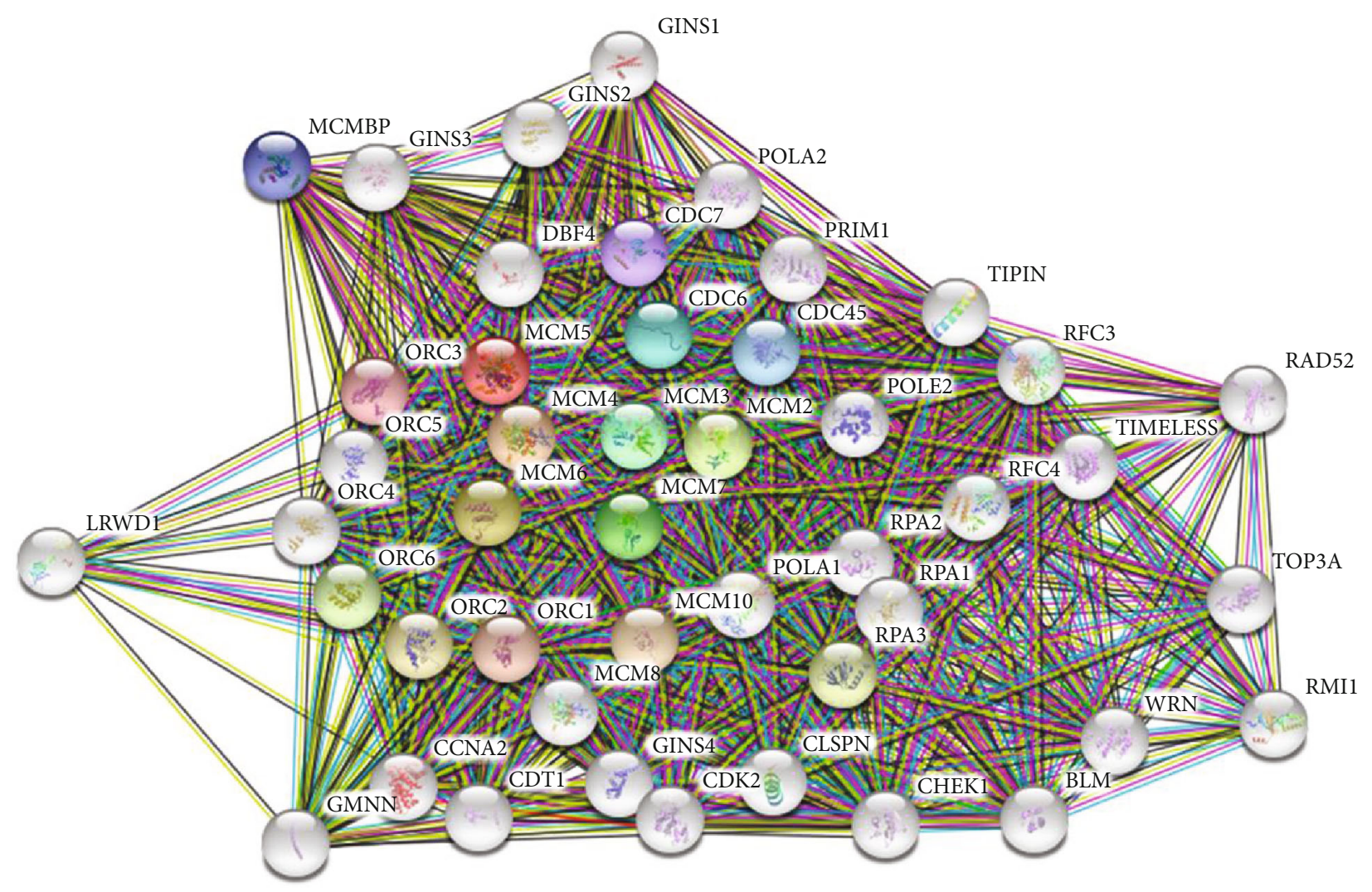

(c)

FIgURE 5: Coexpression and interaction of MCMs at the gene and protein levels in HCC patients. (a) Spearman's correlation analysis of MCMs. (b) The interaction network among MCMs at the gene level based on the GeneMANIA dataset. (c) The interaction network among MCMs at the protein level based on the STRING dataset. 


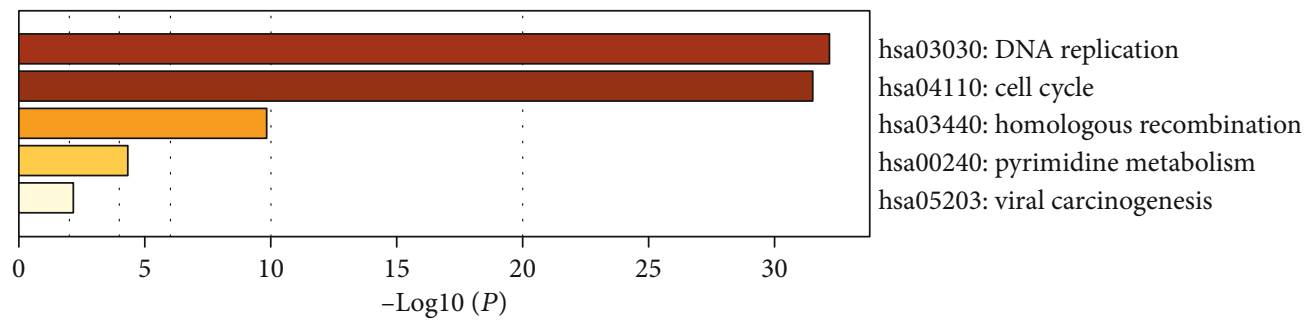

(a)

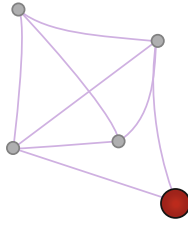

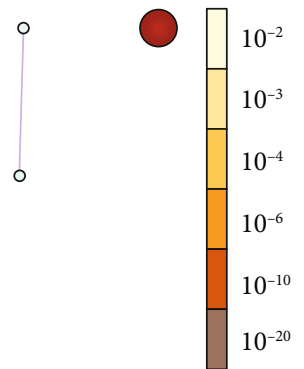

Yheated by

(b)

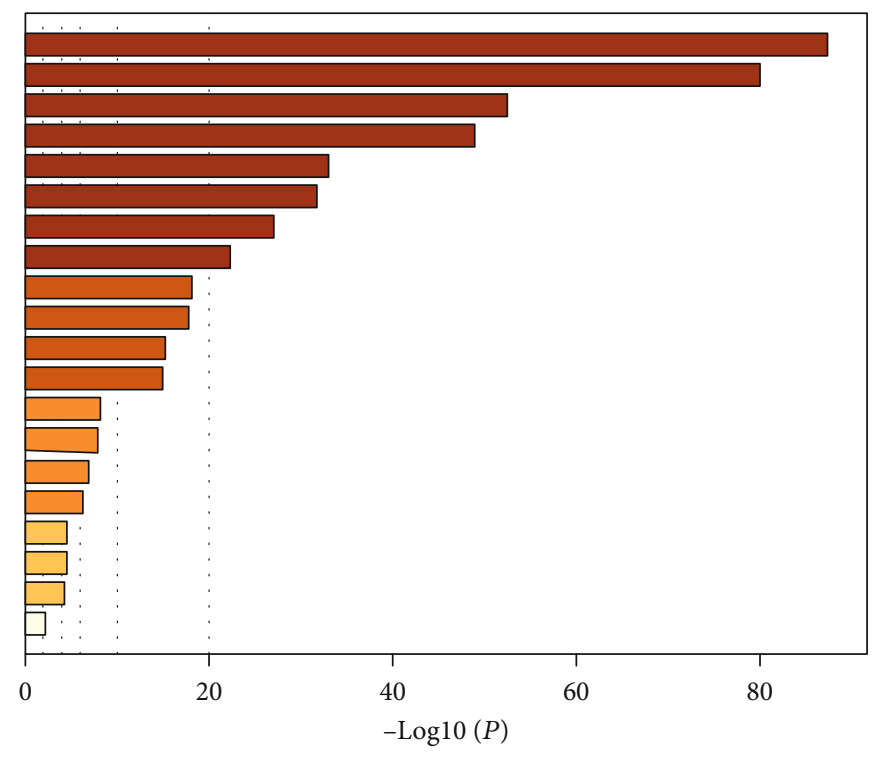

GO:0006260: DNA replication

GO:0006261: DNA-dependent DNA replication

GO:0006270: DNA replication initiation

GO:0033260: nuclear DNA replication

GO:0000724: double-strand break repair via homologous recombination

GO:0005657: replication fork

GO:0000781: chromosome, telomeric region

GO:0006271: DNA strand elongation involved in DNA replication

GO:0032201: telomere maintenance via semi-conservative replication

GO:0006275: regulation of DNA replication

GO:0000076: DNA replication checkpoint

GO:0031298: replication fork protection complex

GO:0071163: DNA replication preinitiation complex assembly

GO:0008156: negative regulation of DNA replication

GO:0000785: chromatin

GO:0034502: protein localization to chromosome

GO:0051304: chromosome separation

GO:0031572: G2 DNA damage checkpoint

GO:0016572: histone phosphorylation

GO:0051347: positive regulation of transferase activity

(c)

Figure 6: Continued. 


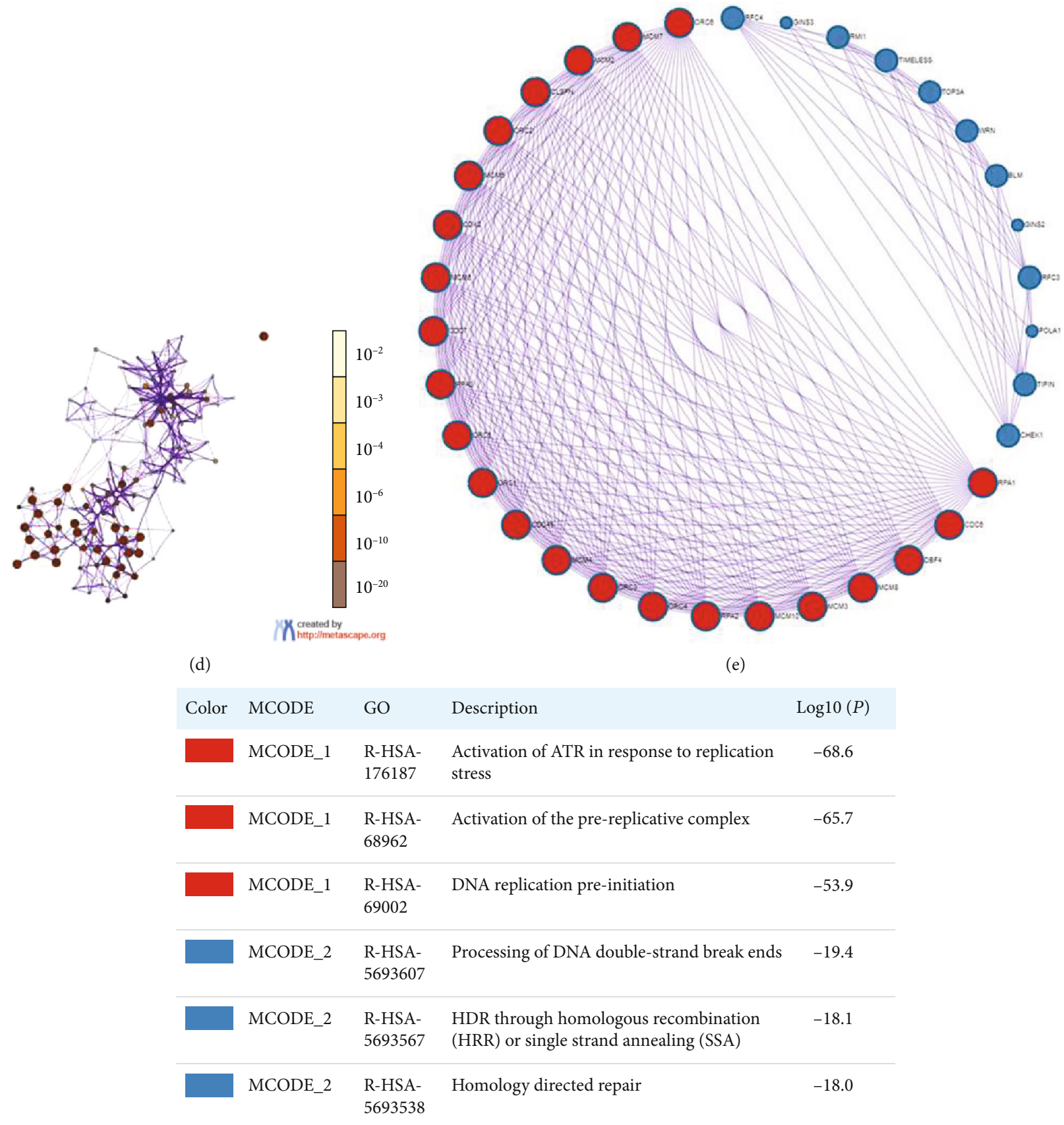

(f)

Figure 6: Gene Ontology (GO) and Kyoto Encyclopedia of Genes and Genomes (KEGG) enrichment analysis of MCMs and neighboring genes in HCC patients. (a) Top 5 KEGG enrichment. (b) Network of KEGG enriched terms. (c) Top 20 GO enrichment. (d) Network of GO enriched terms. (e) Protein-protein interaction (PPI) network by the Metascape database. (f) PPI network by functional enrichment analysis based on MCODE components.

respectively. However, more work and experiments are needed to verify these bioinformatics predictions, which will help to investigate the role of MCM2-7 and related signaling pathways in the development of HCC.

\section{Conclusion}

In this study, we systematically summarized the mRNA and protein level of MCMs and useful prognostic information about MCMs in HCC. Furthermore, we also analyzed the genetic alteration, coexpression, gene/protein network, and GO/KEGG enrichment analysis of MCMs. Relevant results indicated that the mRNA and protein level of MCMs was significantly increased in HCC tissue compared to normal liver tissues. KM plotter analysis showed that high expression of MCMs indicated a worse OS/RFS/PFS/DSS in HCC patients. Importantly, HCC patients with MCM alteration did not display worse OS compared with the ones without MCM 


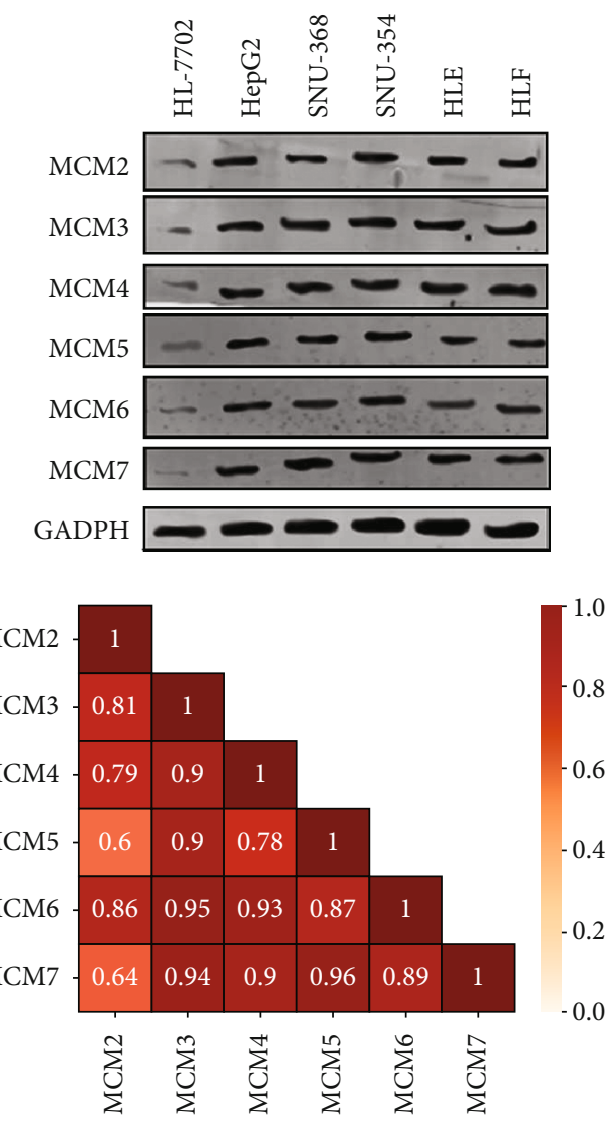

(a)
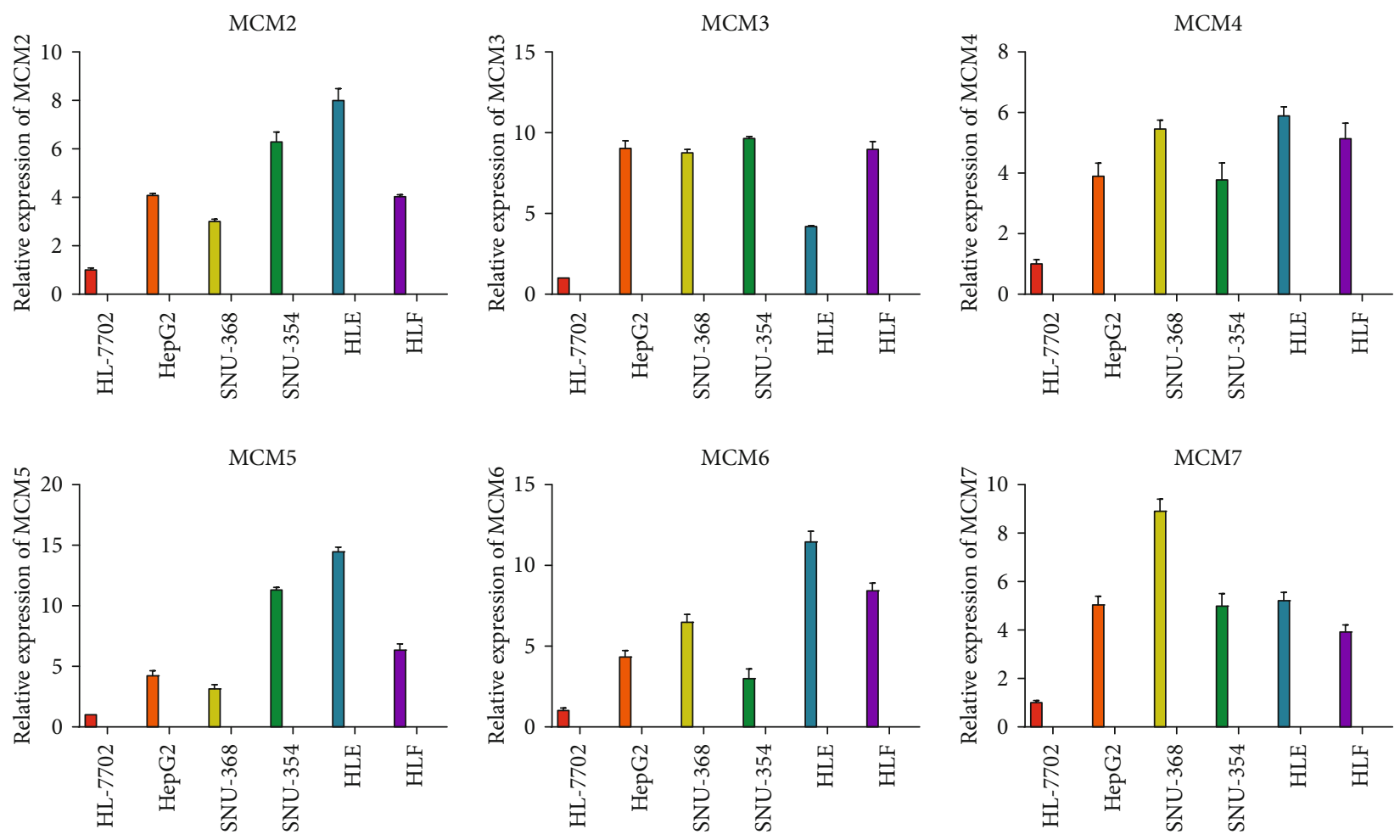

(b)

Figure 7: Continued. 


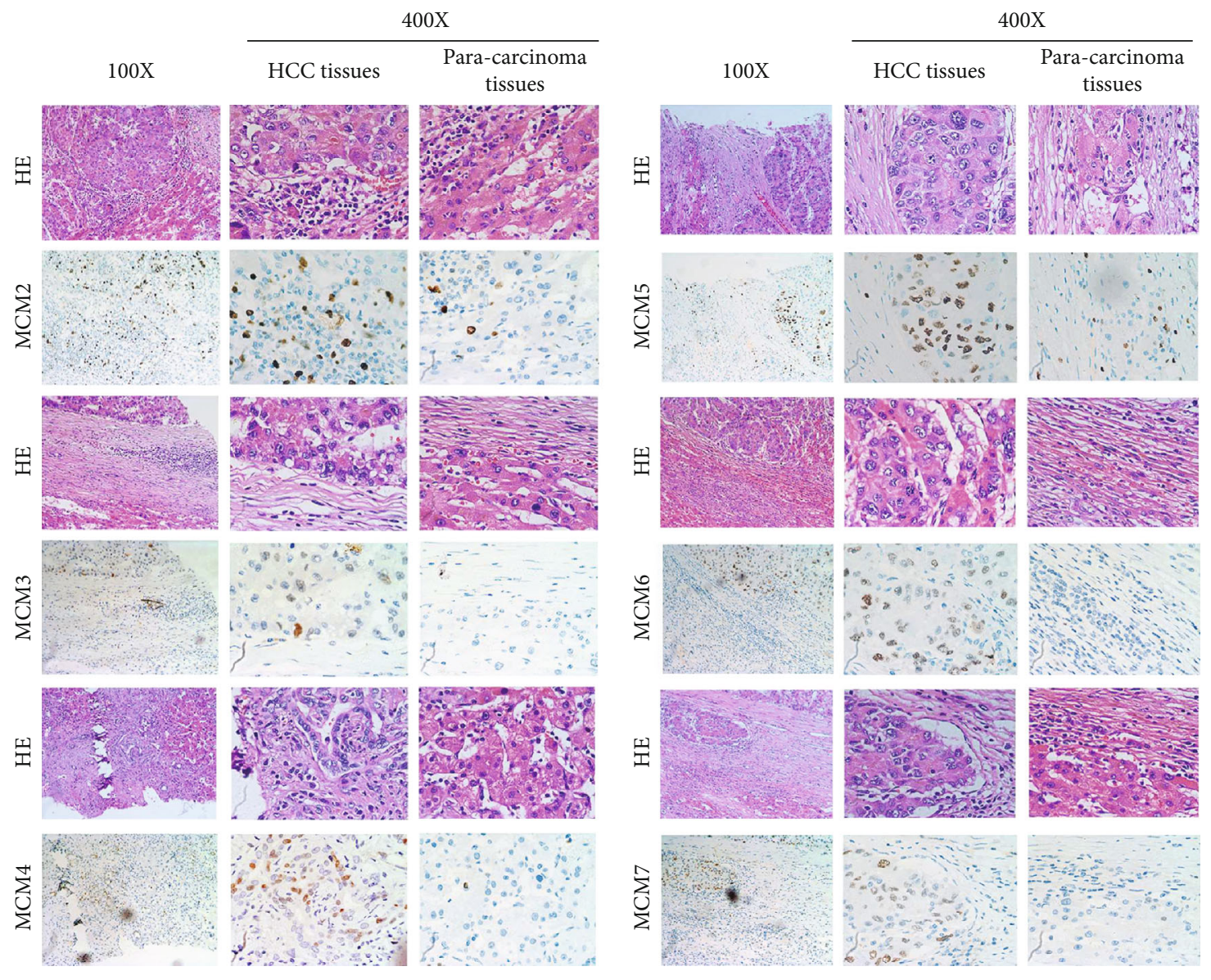

(c)

Figure 7: Identification of MCMs expressed in HCC cell lines. (a) The expression of MCMs in HCC cell lines and normal hepatocyte cell line and the correlation among MCMs in HCC cell lines. (b) The mRNA levels of MCMs in HCC cell lines and normal hepatocyte cell line. (c) The expression of MCMs in HCC tissues. High-magnification microscopic appearances of HCC tissues and paracarcinoma tissue are both taken from the low-magnification microscopic appearance by microscopes.

alteration, which might be attributed to ectopic expression of MCMs mediated by other molecular mechanisms. In conclusion, MCMs could be an effective prognostic marker for HCC. Our results can help to better understand the pathogenesis of HCC and develop more effective clinical treatments in the future.

\section{Data Availability}

The data used to support the findings of this study are available from the corresponding author upon request.

\section{Conflicts of Interest}

The authors declare that they have no conflicts of interest.

\section{Authors' Contributions}

The conception and design were devised by Ting Cao, Shi-jie $\mathrm{Yi}$, and Li-xin Wang. Collection and assembly of data were done by Shi-jie Yi, Juan-xia Zhao, Jiao Xiao, Ni Xie, and Zhi Zeng. Shi-jie Yi, Qi Han, Hai-ou Tang, and Yu-kun Li contributed to the data analysis and interpretation. Yu-kun Li, Shi-jie Yi, and Ting Cao contributed to manuscript writing. Paper revision was done by Juan Zou and Qing Wu. Final approval of the manuscript was done by all the authors. Ting Cao and Shi-jie Yi contributed equally to this work.

\section{Supplementary Materials}

Supplemental Figure S1: the expression of MCMs in different clinical stages. Supplemental Figure S2: the correlation between MCMs. (Supplementary Materials)

\section{References}

[1] R. L. Siegel, K. D. Miller, and A. Jemal, "Cancer statistics, 2018," CA: A Cancer Journal for Clinicians, vol. 68, no. 1, pp. 7-30, 2018.

[2] N. M. Tunissiolli, M. M. U. Castanhole-Nunes, É. C. Pavarino, R. F. da Silva, R. D. C. M. A. da Silva, and E. M. GoloniBertollo, "Clinical, epidemiological and histopathological aspects in patients with hepatocellular carcinoma undergoing 
liver transplantation," Asian Pacific journal of cancer prevention : APJCP, vol. 19, no. 10, pp. 2795-2802, 2018.

[3] E. Wurmbach, Y. B. Chen, G. Khitrov et al., "Genome-wide molecular profiles of $\mathrm{HCV}$-induced dysplasia and hepatocellular carcinoma," Hepatology, vol. 45, no. 4, pp. 938-947, 2007.

[4] J. M. Llovet and M. Beaugrand, "Hepatocellular carcinoma: present status and future prospects," Journal of Hepatology, vol. 38, no. 1, pp. 136-149, 2003.

[5] M. Caselitz, N. Masche, P. Flemming, C. Stern, and S. Kubicka, "Prognosis of hepatocellular carcinoma according to new staging classifications," Deutsche Medizinische Wochenschrift, vol. 129, no. 33, pp. 1725-1730, 2004.

[6] O. Menyhárt, Á. Nagy, and B. Győrffy, "Determining consistent prognostic biomarkers of overall survival and vascular invasion in hepatocellular carcinoma," Royal Society Open Science, vol. 5, no. 12, article 181006, 2018.

[7] X. Wang, T. Yu, X. Liao et al., "The prognostic value of CYP2C subfamily genes in hepatocellular carcinoma," Cancer Medicine, vol. 7, no. 4, pp. 966-980, 2018.

[8] B. He, J. Yin, S. Gong et al., "Bioinformatics analysis of key genes and pathways for hepatocellular carcinoma transformed from cirrhosis," Medicine, vol. 96, no. 25, p. e6938, 2017.

[9] S.-S. Han, Z.-Q. Feng, R. Liu, J. Ye, W.-W. Cheng, and J.-B. Bao, "Bioinformatics analysis and RNA-sequencing of SCAMP3 expression and correlated gene regulation in hepatocellular carcinoma," Oncotargets and Therapy, vol. 13, pp. 1047-1057, 2020.

[10] K. B. Tye, "MCM proteins in DNA replication," Annual Review of Biochemistry, vol. 68, no. 1, pp. 649-686, 1999.

[11] M. Noseda and A. Karsan, "Notch and minichromosome maintenance (MCM) proteins: integration of two ancestral pathways in cell cycle control," Cell Cycle, vol. 5, no. 23, pp. 2704-2709, 2006.

[12] S. Piatti, T. Bohm, J. H. Cocker, J. F. Diffley, and K. Nasmyth, "Activation of S-phase-promoting CDKs in late G1 defines a "point of no return" after which Cdc6 synthesis cannot promote DNA replication in yeast," Genes \& Development, vol. 10, no. 12, pp. 1516-1531, 1996.

[13] M. Noseda, K. Niessen, G. McLean, L. Chang, and A. Karsan, "Notch-dependent cell cycle arrest is associated with downregulation of minichromosome maintenance proteins," Circulation Research, vol. 97, no. 2, pp. 102-104, 2005.

[14] M. Liu, Q. Hu, M. Tu et al., "MCM6 promotes metastasis of hepatocellular carcinoma via MEK/ERK pathway and serves as a novel serum biomarker for early recurrence," Journal of Experimental \& Clinical Cancer Research, vol. 37, no. 1, p. 10, 2018.

[15] C. Pouget, S. Hergalant, E. Lardenois et al., "Ki-67 and MCM6 labeling indices are correlated with overall survival in anaplastic oligodendroglioma, IDH1-mutant and 1p/19q-codeleted: a multicenter study from the French POLA network," Brain Pathology, vol. 30, no. 3, pp. 465-478, 2020.

[16] S. M. Razavi, M. Jafari, M. Heidarpoor, and S. Khalesi, "Minichromosome maintenance-2 (MCM2) expression differentiates oral squamous cell carcinoma from pre-cancerous lesions," vol. 37, no. 3, p. 253, 2015.

[17] M. Liu, J.-S. Li, D.-P. Tian, B. Huang, S. Rosqvist, and M. Su, "MCM2 expression levels predict diagnosis and prognosis in gastric cardiac cancer," Histology \& Histopathology, vol. 28, no. 4, pp. 481-492, 2013.
[18] E. M. Yousef, D. Furrer, D. L. Laperriere et al., "MCM2: an alternative to $\mathrm{Ki}-67$ for measuring breast cancer cell proliferation," Modern Pathology, vol. 30, no. 5, pp. 682-697, 2017.

[19] M. Burger, "MCM2 and MCM5 as prognostic markers in colon cancer: a worthwhile approach," Digestive Diseases and Sciences, vol. 54, no. 2, pp. 197-198, 2009.

[20] H. Li, Z. Lin, Y. Bai et al., "Sinomenine inhibits ovarian cancer cell growth and metastasis by mediating the Wnt $\beta$-catenin pathway via targeting MCM2," Rsc Advances, vol. 7, no. 79, pp. 50017-50026, 2017.

[21] D.-d. Cheng, H.-z. Zhang, J.-q. Yuan, S.-j. Li, Q.-c. Yang, and C.-y. Fan, "Minichromosome maintenance protein 2 and 3 promote osteosarcoma progression via DHX9 and predict poor patient prognosis," Oncotarget, vol. 8, no. 16, pp. 26380-26393, 2017.

[22] C. Yang, Y. Wen, H. Li et al., "Overexpression of minichromosome maintenance 2 predicts poor prognosis in patients with gastric cancer," Oncology Reports, vol. 27, no. 1, pp. 135-142, 2012.

[23] K. Hashimoto, K. Araki, M. Osaki et al., "MCM2 and Ki-67 expression in human lung adenocarcinoma: prognostic implications," Pathobiology, vol. 71, no. 4, pp. 193-200, 2004.

[24] Y. Wang, H. Wang, K. Fu et al., "Cdc7 and Mcm2 expression as markers for the proliferation and prognosis of diffuse large B cell lymphoma," Chinese Journal of Clinical Oncology, vol. 38, no. 18, pp. 1088-1091, 2011.

[25] H. Kato, T. Miyazaki, Y. Fukai et al., "A new proliferation marker, minichromosome maintenance protein 2 , is associated with tumor aggressiveness in esophageal squamous cell carcinoma," Journal of Surgical Oncology, vol. 84, no. 1, pp. 24-30, 2003.

[26] W.-X. Yang, Y.-Y. Pan, and C.-G. You, “CDK1, CCNB1, CDC20, BUB1, MAD2L1, MCM3, BUB1B, MCM2, and RFC4 may be potential therapeutic targets for hepatocellular carcinoma using integrated bioinformatic analysis," BioMed Research International, vol. 2019, Article ID 1245072, 16 pages, 2019.

[27] M. Deng, J. Sun, S. Xie et al., "Inhibition of MCM2 enhances the sensitivity of ovarian cancer cell to carboplatin," Molecular Medicine Reports, vol. 20, no. 3, pp. 2258-2266, 2019.

[28] Z. J. Ashkavandi, A. D. Najvani, A. A. Tadbir, S. Pardis, M. A. Ranjbar, and M. J. Ashraf, "MCM3 as a novel diagnostic marker in benign and malignant salivary gland tumors," Asian Pacific Journal of Cancer Prevention, vol. 14, no. 6, pp. 34793482, 2013.

[29] C. Hua, G. Zhao, Y. Li, and L. Bie, "Minichromosome maintenance (MCM) family as potential diagnostic and prognostic tumor markers for human gliomas," Bmc Cancer, vol. 14, no. 1, 2014.

[30] S.-A. Ha, S. M. Shin, H. Namkoong et al., "Cancer-associated expression of minichromosome maintenance 3 gene in several human cancers and its involvement in tumorigenesis," Clinical Cancer Research, vol. 10, no. 24, pp. 8386-8395, 2004.

[31] X.-P. Huang, T.-H. Rong, Q.-L. Wu et al., "MCM4 expression in esophageal cancer from southern China and its clinical significance," Journal of Cancer Research \& Clinical Oncology, vol. 131, no. 10, pp. 677-682, 2005.

[32] L. P. Chen, R. H. Liu, and F. X. Zhao, "Expression of MCM4 and CDC6 in uterine cervical carcinoma and its relation with HPV16/18 infection," Chinese Journal of Cancer Biotherapy, vol. 15, no. 4, pp. 374-378, 2008. 
[33] J. Kikuchi, I. Kinoshita, Y. Shimizu et al., "Minichromosome maintenance (MCM) protein 4 as a marker for proliferation and its clinical and clinicopathological significance in nonsmall cell lung cancer," Lung Cancer, vol. 72, no. 2, pp. 229237, 2011.

[34] S. Y. Yu, Y.-P. Wang, J. Y.-F. Chang, W.-R. Shen, H.-M. Chen, and C.-P. Chiang, "Increased expression of MCM5 is significantly associated with aggressive progression and poor prognosis of oral squamous cell carcinoma," Journal of Oral Pathology \& Medicine, vol. 43, no. 5, pp. 344-349, 2014.

[35] N. Murphy, "p16INK4A, CDC6, and MCM5: predictive biomarkers in cervical preinvasive neoplasia and cervical cancer," Journal of Clinical Pathology, vol. 58, no. 5, pp. 525-534, 2005.

[36] C. Mio, E. Lavarone, K. Conzatti et al., "MCM5 as a target of BET inhibitors in thyroid cancer cells," Endocrine Related Cancer, vol. 23, no. 4, pp. 335-347, 2016.

[37] A. S. Brems-Eskildsen, K. Zieger, H. Toldbod et al., "Prediction and diagnosis of bladder cancer recurrence based on urinary content of hTERT, SENP1, PPP1CA, and MCM5 transcriptsvol. 10, no. 1, p. 646.

[38] A. Hendricks, F. Gieseler, S. Nazzal et al., "Prognostic relevance of topoisomerase II $\alpha$ and minichromosome maintenance protein 6 expression in colorectal cancer," $B M C$ Cancer, vol. 19, no. 1, p. 429, 2019.

[39] M. S. M. Issac, E. Yousef, M. R. Tahir, and L. A. Gaboury, "MCM2, MCM4, and MCM6 in breast cancer: clinical utility in diagnosis and prognosis," Neoplasia, vol. 21, no. 10, pp. 1015-1035, 2019.

[40] J. Hotton, M. Agopiantz, A. Leroux et al., "Minichromosome maintenance complex component 6 (MCM6) expression correlates with histological grade and survival in endometrioid endometrial adenocarcinoma," Virchows Archiv, vol. 472, no. 4, pp. 623-633, 2018.

[41] C. Vigouroux, J. M. Casse, S. F. Battaglia-Hsu, L. Brochin, and G. Gauchotte, "Methyl (R217) HuR and MCM6 are inversely correlated and are prognostic markers in non small cell lung carcinoma," Lung Cancer, vol. 89, no. 2, pp. 189-196, 2015.

[42] G. Gauchotte, C. Vigouroux, F. Rech et al., "Expression of minichromosome maintenance MCM6 protein in meningiomas is strongly correlated with histologic grade and clinical outcome," The American Journal of Surgical Pathology, vol. 36, no. 2, pp. 283-291, 2012.

[43] D. Henderson, L. Hall, N. Prpic et al., "The selection and characterization of antibodies to minichromosome maintenance proteins that highlight cervical dysplasia," Journal of Immunological Methods, vol. 370, no. 1-2, pp. 1-13, 2011.

[44] S. Karimi, F. Mohammadi, K. Khodadad, H. Emami, and L. Seyfollahi, "High expression of minichromosome maintenance protein 6 in classic Hodgkin's lymphoma points to a cell cycle arrest in G1 phase," Archives of Iranian Medicine., vol. 11, no. 5, pp. 532-538, 2008.

[45] T. Gambichler, A. Breininger, S. Rotterdam, P. Altmeyer, M. Stücker, and A. Kreuter, "Expression of minichromosome maintenance proteins in Merkel cell carcinoma," Journal of the European Academy of Dermatology \& Venereology Jeadv., vol. 23, no. 10, pp. 1184-1188, 2009.

[46] V. Padmanabhan, "DNA replication regulation protein $\mathrm{Mcm} 7$ as a marker of proliferation in prostate cancer," Journal of Clinical Pathology, vol. 57, no. 10, pp. 1057-1062, 2004.

[47] K. Qu, Z. Wang, H. Fan et al., "MCM7 promotes cancer progression through cyclin D1-dependent signaling and serves as a prognostic marker for patients with hepatocellular carcinoma," Cell Death \& Disease, vol. 8, no. 2, p. e2603, 2017.

[48] W. B. Dai, P. Huang, and Z. P. Ren, "Relationship between expression of MCM7 and Ki-67 and response to neoadjuvant chemotherapy in breast cancer," Journal of Practical Oncology, vol. 25, no. 1, pp. 20-23, 2010.

[49] J. S. Lee, H. S. Cheong, Y. Koh, K.-S. Ahn, H. D. Shin, and S.S. Yoon, "MCM7 polymorphisms associated with the AML relapse and overall survival," Annals of Hematology, vol. 96, no. 1, pp. 93-98, 2017.

[50] D. R. Rhodes, J. Yu, K. Shanker et al., "ONCOMINE: a cancer microarray database and integrated data-mining platform," Neoplasia, vol. 6, no. 1, pp. 1-6, 2004.

[51] Z. Tang, C. Li, B. Kang, G. Gao, C. Li, and Z. Zhang, "GEPIA: a web server for cancer and normal gene expression profiling and interactive analyses," Nucleic Acids Research, vol. 45, no. W1, pp. W98-W102, 2017.

[52] S. Mostafavi, D. Ray, D. Warde-Farley, C. Grouios, and Q. Morris, "GeneMANIA: a real-time multiple association network integration algorithm for predicting gene function," Genome Biology, vol. 9, Supplement 1, p. S4, 2008.

[53] D. Szklarczyk, J. H. Morris, H. Cook et al., "The STRING database in 2017: quality-controlled protein-protein association networks, made broadly accessible," Nucleic Acids Research, vol. 45, no. D1, pp. D362-D368, 2017.

[54] J. Gao, B. A. Aksoy, U. Dogrusoz et al., "Integrative analysis of complex cancer genomics and clinical profiles using the cBioPortal," Science Signaling, vol. 6, no. 269, p. pl1, 2013.

[55] D. Warde-Farley, S. L. Donaldson, O. Comes et al., "The GeneMANIA prediction server: biological network integration for gene prioritization and predicting gene function," Nucleic Acids Research, vol. 38, supplement 2, pp. W214W220, 2010.

[56] J. Zou, H. Li, Q. Huang et al., "Dopamine-induced SULT1A3/4 promotes EMT and cancer stemness in hepatocellular carcinoma," Tumour biology : the journal of the International Society for Oncodevelopmental Biology and Medicine, vol. 39, no. 10, 2017.

[57] X. Chen, S. T. Cheung, S. So et al., "Gene expression patterns in human liver cancers," Molecular Biology of the Cell, vol. 13, no. 6, pp. 1929-1939, 2002.

[58] S. Roessler, H.-L. Jia, A. Budhu et al., "A unique metastasis gene signature enables prediction of tumor relapse in earlystage hepatocellular carcinoma patients," Cancer Research, vol. 70, no. 24, pp. 10202-10212, 2010.

[59] H. Xie, C. Hua, L. Sun et al., " $17 \beta$-estradiol induces CD40 expression in dendritic cells via MAPK signaling pathways in a minichromosome maintenance protein 6-dependent mannervol. 63, no. 8, pp. 2425-2435.

[60] A. Quaglia, M. Mcstay, K. Stoeber et al., "Novel markers of cell kinetics to evaluate progression from cirrhosis to hepatocellular carcinoma," Liver International., vol. 26, no. 4, pp. 424432, 2006.

[61] G. Kaur, S. D. Balasubramaniam, Y. J. Lee, V. Balakrishnan, and C. E. Oon, "Minichromosome maintenance complex (MCM) genes profiling and MCM2 protein expression in cervical cancer development," Asian Pacific Journal of Cancer Prevention, vol. 20, no. 10, pp. 3043-3049, 2019.

[62] Y. Zhao, Y. Wang, F. Zhu, J. Zhang, X. Ma, and D. Zhang, "Gene expression profiling revealed MCM3 to be a better marker than $\mathrm{Ki} 67$ in prognosis of invasive ductal breast 
carcinoma patients," Clinical and experimental medicine, vol. 20, no. 2, pp. 249-259, 2020.

[63] B. Choy, A. LaLonde, J. Que, T. Wu, and Z. Zhou, "MCM4 and MCM7, potential novel proliferation markers, significantly correlated with Ki-67, Bmil, and cyclin E expression in esophageal adenocarcinoma, squamous cell carcinoma, and precancerous lesions," Human Pathology, vol. 57, pp. 126-135, 2016.

[64] B. Gong, M. Ma, X. Yang, W. Xie, Y. Luo, and T. Sun, "MCM5 promotes tumour proliferation and correlates with the progression and prognosis of renal cell carcinoma," International urology and nephrology., vol. 51, no. 9, pp. 1517-1526, 2019.

[65] B. Choy, A. LaLonde, J. Que, T. Wu, and Z. Zhou, "MCM4 and MCM7, potential novel proliferation markers, significantly correlated with Ki67, Bmil, and cyclin E expression in esophageal adenocarcinoma, squamous cell carcinoma, and precancerous lesions," Human Pathology, vol. 57, pp. 126-135, 2016.

[66] T. Scholzen and J. Gerdes, "The Ki-67 protein: from the known and the unknown," Journal of Cellular Physiology., vol. 182, no. 3, pp. 311-322, 2000.

[67] P. Wen, T. Chidanguro, Z. Shi et al., "Identification of candidate biomarkers and pathways associated with SCLC by bioinformatics analysis," Molecular Medicine Reports, vol. 18, no. 2, pp. 1538-1550, 2018.

[68] C.-Y. Lin, H.-Y. Wu, Y.-L. Hsu et al., "Suppression of drugresistant non-small-cell lung cancer with inhibitors targeting minichromosomal maintenance protein," Journal of Medicinal Chemistry, vol. 63, no. 6, pp. 3172-3187, 2020.

[69] M. Tarsounas and P. Sung, "The antitumorigenic roles of BRCA1-BARD1 in DNA repair and replication," Nature Reviews Molecular cell Biology, vol. 21, no. 5, pp. 284-299, 2020.

[70] S. R. Sweeney, A. Kavanaugh, A. Lodi et al., "Metabolomic profiling predicts outcome of rituximab therapy in rheumatoid arthritis," RMD Open, vol. 2, no. 2, article e000289, 2016.

[71] J. Simon, Z. Liu, N. Brenner et al., "Validation of an EpsteinBarr Virus antibody risk stratification signature for nasopharyngeal carcinoma by Use of multiplex serology," Journal of Clinical Microbiology, vol. 58, no. 5, 2020.

[72] S. Z. Yu, "Facilitating the continuous decline on the incidence rates of hepatitis $B$ and liver cancer through cutting off the mother-to-child viral transmission, based on the "prevention first" strategy, in China," Zhonghua liu xing bing xue za zhi = Zhonghua liuxingbingxue zazhi, vol. 40, no. 12, pp. 16501653, 2019. 